Article

\title{
Genetic Diversity and Combining Ability of White Maize Inbred Lines under Different Plant Densities
}

\author{
Mohamed M. Kamara ${ }^{1}$ (), Medhat Rehan ${ }^{2,3}$, Khaled M. Ibrahim ${ }^{4}$, Abdullah S. Alsohim ${ }^{3}$, \\ Mohsen M. Elsharkawy ${ }^{5}\left(\mathbb{D}\right.$, Ahmed M. S. Kheir ${ }^{6} \mathbb{D}^{\mathbb{D}}$, Emad M. Hafez ${ }^{1}$ (D) and \\ Mohamed A. El-Esawi ${ }^{7, *(D)}$ \\ 1 Department of Agronomy, Faculty of Agriculture, Kafrelsheikh University, Kafr El-Sheikh 33516, Egypt; \\ mohamed.kamara@agr.kfs.edu.eg (M.M.K.); emadhafez2012@agr.kfs.edu.eg (E.M.H.) \\ 2 Department of Genetics, Faculty of Agriculture, Kafrelsheikh University, Kafr El-Sheikh 33516, Egypt; \\ medhat.rehan@agr.kfs.edu.eg \\ 3 Department of Plant Production and Protection, College of Agriculture and Veterinary Medicine, Qassim \\ University, Burydah 51452, Saudi Arabia; a.alsohim@qu.edu.sa \\ 4 Agronomy Department, Faculty of Agriculture, New Valley University, El-Kharga 72511, Egypt; \\ kh_ibrahim75@aun.edu.eg \\ 5 Department of Agricultural Botany, Faculty of Agriculture, Kafrelsheikh University, \\ Kafr Elsheikh 33516, Egypt; mohsen.abdelrahman@agr.kfs.edu.eg \\ 6 Soils, Water and Environment Research Institute, Agricultural Research Center, Giza 12112, Egypt; \\ ahmed.kheir@arc.sci.eg \\ 7 Botany Department, Faculty of Science, Tanta University, Tanta 31527, Egypt \\ * Correspondence: mohamed.elesawi@science.tanta.edu.eg
}

Received: 7 August 2020; Accepted: 31 August 2020; Published: 3 September 2020

\begin{abstract}
Knowledge of combining ability and genetic diversity are important prerequisites for the development of outstanding hybrids that are tolerant to high plant density. This work was carried out to assess general combining ability (GCA) and specific combining ability (SCA), identify promising hybrids, estimate genetic diversity among the inbred lines and correlate genetic distance to hybrid performance and SCA across different plant densities. A total of $28 \mathrm{~F}_{1}$ hybrids obtained by crossing eight adverse inbred lines (four local and four exotic) were evaluated under three plant densities 59,500 (D1), 71,400 (D2) and 83,300 (D3) plants ha ${ }^{-1}$ using spilt plot design with three replications at two locations during 2018 season. Increasing plant density from D1 to D3 significantly decreased leaf angle (LANG), chlorophyll content (CHLC), all ear characteristics and grain yield per plant (GYPP). Contrarily, days to silking (DTS), anthesis-silking interval (ASI), plant height (PLHT), ear height (EHT), and grain yield per hectare (GYPH) were significantly increased. Both additive and non-additive gene actions were involved in the inheritance of all the evaluated traits, but additive gene action was predominant for most traits. Inbred lines $\mathrm{L}_{1}, \mathrm{~L}_{2}$, and $\mathrm{L}_{5}$ were the best general combiners for increasing grain yield and other desirable traits across research environments. Two hybrids $\mathrm{L}_{2} \times \mathrm{L}_{5}$ and $\mathrm{L}_{2} \times \mathrm{L}_{8}$ were found to be good specific combiners for ASI, LANG, GYPP and GYPH. Furthermore, these hybrids are ideal for further testing and promotion for commercialization under high plant density. Genetic distance (GD) among pairs of inbred lines ranged from 0.31 to 0.78 , with an average of 0.61 . Clustering based on molecular GD has effectively grouped the inbred lines according to their origin. No significant correlation was found between GD and both hybrid performance and SCA for grain yield and other traits and proved to be of no predictive value. Nevertheless, SCA could be used to predict the hybrid performance across all plant densities. Overall, this work presents useful information regarding the inheritance of maize grain yield and other important traits under high plant density.
\end{abstract}

Keywords: maize; density tolerance; combining ability; gene effects; genetic diversity 


\section{Introduction}

Maize (Zea mays L.) is one of the main economic crops that subsidize global food security. It is widely used for food, animal feed, edible oil and fuel worldwide [1]. In Egypt, maize is considered the second most important crop, with the annual production of the grain reaching about 7.30 Mt from approximately 0.94 Mha in 2018 [2]. This production is insufficient to meet the demands of a fast-growing population. The gap between production and consumption is approximately $45 \%$ [3]. This gap could be narrowed by further increase in the hybrids yield potential and total yield production from unit area. [4]. Increasing planting density is required to increase grain yield production in maize [5]. The average density of intense maize cultivation in the USA is 97,500 plants ha $^{-1}$ [6]. The recommended planting density in Egypt is 53,533 plants ha ${ }^{-1}$ [7], which is around half the amount used in the USA. The use of lower plant densities decreases light interception, leading to high grain production per plant but low grain production per unit area [8]. The yield production could be maximized by growing maize hybrids that can tolerate high plant density up to 100,000 plants ha ${ }^{-1}$. However, high plant densities enhance interplant competition for light, nutrients, and water [9]. Additionally, it increases the anthesis-silking interval [10], thereby increasing kernel abortion [11] and reducing single plant yield. Al-Naggar et al. [12] showed that with increased planting density, plant and ear heights increased, whereas chlorophyll content, grains per ear and thousand grain weights decreased. The tolerance of the current Egyptian maize hybrids to high plant densities is low. This probably attributed to their tallness, decumbent leaf, one-eared and large size $[7,13]$. Conversely, modern maize hybrids in developed countries are characterized by early silking, short anthesis-silking interval and prolificacy, which are essential adaptive traits to high plant density tolerance $[10,14-16]$.

Breeding programs should be directed towards the development of hybrids that are not only high yielding, but also show enhanced adaptability to high plant density tolerance. The successful identification of desirable hybrid combinations depends on the combining ability of the parents and the gene effects involved in the expression of target trait [17]. Furthermore, knowledge of gene action is important to devise an appropriate breeding strategy [18]. General combining ability (GCA) and specific combining ability (SCA) are widely used in selection of good parents and hybrids, respectively [19]. Among different biometrical approaches, the diallel mating design is commonly used by maize breeders to estimate GCA and SCA effects [20-22]. GCA is associated with additive gene effects, whereas SCA is typically associated with non-additive gene effects [23]. Both additive and non-additive gene actions were reported to be important in the inheritance of maize grain yield under high plant density [24]. However, the grain yield and other assessed traits under different plant densities among selected maize inbred lines were mostly controlled by additive gene action $[7,25]$.

The assessment of the diversity and genetic distance in the available maize inbreds is important for a hybrid breeding program, in order to identify inbreds that would produce crosses with good levels of heterosis without testing all hybrids combinations [26,27]. Different types of DNA markers are available to estimate genetic distance. The simple sequence repeat (SSR) markers or microsatellites have been considered as the markers of choice owing to their co-dominant, high polymorphic, multi-allelic nature and high reproducibility [28-30]. However, contradictory results have been reported with respect to the relationship between genetic distance and hybrid performance in maize. Significant correlations were reported between molecular marker-based GD and $\mathrm{F}_{1}$ hybrid grain yield in maize [31,32]. Whereas, other studies reported no significant correlation [33,34]. The objectives of this study were (i) to estimate GCA of the inbred lines and SCA of the hybrids under different plant densities; (ii) to determine the mode of gene action controlling grain yield and other important agronomic traits; (iii) to identify promising hybrids that yield well at high plant density; and (iv) to assess genetic diversity among the eight inbred lines and correlate genetic distance to hybrid performance and SCA. 


\section{Results}

\subsection{Analysis of Variance}

The analysis of variance (ANOVA) revealed highly significant mean squares for locations (L), densities (D), hybrids $(\mathrm{H})$ and their interactions $(\mathrm{L} \times \mathrm{D}, \mathrm{H} \times \mathrm{L}, \mathrm{H} \times \mathrm{D}$ and $\mathrm{H} \times \mathrm{D} \times \mathrm{L})$ for all the studied characteristics (Table 1). Moreover, general combining ability (GCA) and specific combining ability (SCA) mean squares were highly significant for all the measured traits. The magnitude of GCA mean squares was higher than that of SCA mean squares (the ratio of GCA/SCA was higher than the unity) for all the studied traits, except number of kernels per row (NKPR) trait. Significant mean squares of $\mathrm{GCA} \times \mathrm{L}, \mathrm{SCA} \times \mathrm{L}, \mathrm{GCA} \times \mathrm{D}, \mathrm{SCA} \times \mathrm{D}, \mathrm{GCA} \times \mathrm{L} \times \mathrm{D}, \mathrm{SCA} \times \mathrm{L} \times \mathrm{D}$ interactions were detected for all the studied traits, except GCA $\times$ L and GCA $\times$ L $\times$ D for leaf angle (LANG) and chlorophyll content $(\mathrm{CHLC}), \mathrm{GCA} \times \mathrm{D}$ for ear diameter $(\mathrm{ED})$ and $\mathrm{SCA} \times \mathrm{D}$ for EHT, LANG and ED were not significant.

Table 1. Analysis of variance for the evaluated crosses under three plant densities combined across two locations for all the studied traits.

\begin{tabular}{|c|c|c|c|c|c|c|c|}
\hline SOV & DF & DTS & ASI & PLHT & EHT & LANG & CHLC \\
\hline Locations (L) & 1 & $1114.26^{* *}$ & $12.87 * *$ & $16,592.96^{* *}$ & $6489.21 * *$ & 108.64 * & $400.29 * *$ \\
\hline $\operatorname{Rep}(\mathrm{L})$ & 4 & 15.14 & 0.58 & 325.06 & 138.65 & 14.87 & 10.84 \\
\hline Densities (D) & 2 & $1899.48^{* *}$ & $73.14^{* *}$ & $23,422.30^{* *}$ & $9384.04 * *$ & $603.65^{* *}$ & $1585.67^{* *}$ \\
\hline $\mathrm{L} \times \mathrm{D}$ & 2 & $308.23^{* *}$ & $22.39 * *$ & $9852.25^{* *}$ & $5708.38^{* *}$ & $27.17^{* *}$ & $180.79^{* *}$ \\
\hline Error a & 8 & 1.07 & 0.19 & 121.77 & 53.15 & 3.33 & 2.34 \\
\hline Hybrids (H) & 27 & $28.04^{* *}$ & $7.89^{* *}$ & $6842.02 * *$ & $2056.81^{* *}$ & $425.82 * *$ & $119.32 * *$ \\
\hline GCA & 7 & $57.16^{* *}$ & $12.03^{* *}$ & $11,397.27^{* *}$ & $2447.44^{* *}$ & $836.14^{* *}$ & $162.00 * *$ \\
\hline SCA & 20 & $17.85^{* *}$ & $6.44^{* *}$ & $5247.69 * *$ & $1920.08^{* *}$ & $282.20 * *$ & $104.38^{* *}$ \\
\hline $\mathrm{H} \times \mathrm{L}$ & 27 & $61.66^{* *}$ & $0.83^{* *}$ & $796.40^{* *}$ & $362.93^{* *}$ & $4.15^{* *}$ & $3.61^{* *}$ \\
\hline $\mathrm{GCA} \times \mathrm{L}$ & 7 & $52.48^{* *}$ & $0.88^{* *}$ & $915.53 * *$ & $309.35^{* *}$ & 3.16 & 3.57 \\
\hline $\mathrm{SCA} \times \mathrm{L}$ & 20 & $64.88^{* *}$ & $0.81^{* *}$ & $754.71 * *$ & $381.68^{* *}$ & $4.49^{* *}$ & $3.62 *$ \\
\hline $\mathrm{H} \times \mathrm{D}$ & 54 & $5.01 * *$ & $0.31 * *$ & $254.88^{* *}$ & $60.51^{* *}$ & $4.57^{* *}$ & $10.19^{* *}$ \\
\hline $\mathrm{GCA} \times \mathrm{D}$ & 14 & $4.54^{* *}$ & $0.30 * *$ & 212.94 ** & $78.99 *$ & $6.63^{* *}$ & $19.13^{* *}$ \\
\hline $\mathrm{SCA} \times \mathrm{D}$ & 40 & $5.18^{* *}$ & $0.31 * *$ & $269.56^{* *}$ & 54.03 & 3.85 & $7.06^{* *}$ \\
\hline $\mathrm{H} \times \mathrm{D} \times \mathrm{L}$ & 54 & $63.25^{* *}$ & $0.88^{* *}$ & 592.24 ** & $397.54^{* *}$ & 4.11 * & $4.30^{* *}$ \\
\hline $\mathrm{GCA} \times \mathrm{L} \times \mathrm{D}$ & 14 & $64.03 * *$ & $0.86^{* *}$ & $544.25^{* *}$ & $363.40^{* *}$ & 3.90 & 2.64 \\
\hline $\mathrm{SCA} \times \mathrm{L} \times \mathrm{D}$ & 40 & $62.97^{* *}$ & $0.88^{* *}$ & $609.03^{* *}$ & $409.48 * *$ & $4.19 *$ & $4.88^{* *}$ \\
\hline Error b & 324 & 0.81 & 0.14 & 84.07 & 42.33 & 2.69 & 1.93 \\
\hline GCA/SCA & & 3.20 & 1.87 & 2.17 & 1.27 & 2.96 & 1.55 \\
\hline SOV & DF & ED & NRPE & NKPR & TKW & GYPP & GYPH \\
\hline Locations (L) & 1 & $2.09 *$ & $36.35 * *$ & $353.21 * *$ & $8232.88^{* *}$ & $12,079.40 * *$ & $40.40 * *$ \\
\hline $\operatorname{Rep}(\mathrm{L})$ & 4 & 0.26 & 1.35 & 12.53 & 225.52 & 285.31 & 0.78 \\
\hline Densities (D) & 2 & $21.93^{* *}$ & $88.74^{* *}$ & $2229.25^{* *}$ & $91,176.13^{* *}$ & $50,563.13^{* *}$ & $58.38^{* *}$ \\
\hline $\mathrm{L} \times \mathrm{D}$ & 2 & $1.78^{* *}$ & $6.33^{* *}$ & $331.20 * *$ & $3151.63 * *$ & $14,971.59 * *$ & $54.39 * *$ \\
\hline Error a & 8 & 0.18 & 0.52 & 3.09 & 192.11 & 56.68 & 0.30 \\
\hline Hybrids (H) & 27 & $1.01^{* *}$ & $8.75^{* *}$ & $56.88^{* *}$ & $10,944.20^{* *}$ & $9941.33^{* *}$ & $49.21 * *$ \\
\hline GCA & 7 & $1.07^{* *}$ & $16.50^{* *}$ & $41.59 * *$ & $12,835.71^{* *}$ & $13,527.67^{* *}$ & $67.17^{* *}$ \\
\hline SCA & 20 & $0.99^{* *}$ & $6.04^{* *}$ & $62.24^{* *}$ & $10,282.17^{* *}$ & $8686.11^{* *}$ & $42.93 * *$ \\
\hline $\mathrm{H} \times \mathrm{L}$ & 27 & $1.25 * *$ & $18.90^{* *}$ & $21.01^{* *}$ & $2126.28^{* *}$ & $1230.60^{* *}$ & $4.24^{* *}$ \\
\hline $\mathrm{GCA} \times \mathrm{L}$ & 7 & $1.56^{* *}$ & $20.27^{* *}$ & $17.14^{* *}$ & $2328.04^{* *}$ & $1621.69 * *$ & $5.61 * *$ \\
\hline $\mathrm{SCA} \times \mathrm{L}$ & 20 & $1.14^{* *}$ & $18.42 * *$ & $22.36^{* *}$ & $2055.67^{* *}$ & $1093.72 * *$ & $3.75^{* *}$ \\
\hline $\mathrm{H} \times \mathrm{D}$ & 54 & $0.20^{* *}$ & $0.98^{* *}$ & $8.77^{* *}$ & $360.55^{* *}$ & $187.93^{* *}$ & $0.71^{* *}$ \\
\hline $\mathrm{GCA} \times \mathrm{D}$ & 14 & 0.24 & $1.45^{* *}$ & $10.49 * *$ & $560.46^{* *}$ & $166.93^{* *}$ & $0.73^{* *}$ \\
\hline $\mathrm{SCA} \times \mathrm{D}$ & 40 & 0.19 & $0.82 * *$ & $8.17^{* *}$ & $290.58 * *$ & $195.28 * *$ & $0.70 * *$ \\
\hline $\mathrm{H} \times \mathrm{D} \times \mathrm{L}$ & 54 & $1.27^{* *}$ & $13.20 * *$ & $16.84^{* *}$ & $621.87 * *$ & $1517.64^{* *}$ & $5.45^{* *}$ \\
\hline $\mathrm{GCA} \times \mathrm{L} \times \mathrm{D}$ & 14 & $1.45^{* *}$ & $11.20 * *$ & $19.16^{* *}$ & $529.08 * *$ & $1951.52 * *$ & $6.99 * *$ \\
\hline $\mathrm{SCA} \times \mathrm{L} \times \mathrm{D}$ & 40 & $1.21^{* *}$ & $13.89 * *$ & $16.02 * *$ & $654.34 * *$ & $1365.78^{* *}$ & $4.91^{* *}$ \\
\hline Error b & 324 & 0.15 & 0.38 & 2.41 & 143.75 & 45.22 & 0.25 \\
\hline GCA/SCA & & 1.08 & 2.73 & 0.67 & 1.25 & 1.56 & 1.57 \\
\hline
\end{tabular}

${ }^{*}$ and ${ }^{* *}$ significant at 0.05 and 0.01 levels of probability, respectively. DTS: days to $50 \%$ silking, ASI: anthesis-silking interval, PLHT: plant height, EHT: ear height, LANG: leaf angle, CHLC: chlorophyll content, ED: ear diameter, NRPE: number of rows per ear, NKPR: number of kernels per row, TKW: thousand kernel weight, GYPP: grain yield per plant and GYPH: grain yield per hectare. 


\subsection{Changes in the Studied Traits Due to Increased Plant Density}

Across the two locations, the mean of grain yield per plant (GYPP) was significantly decreased as plant density increased from D1 to D2 and D3 by -9.60 and $-20.59 \%$, respectively, as compared to D1 (Figure 1A). This reduction was accompanied by reductions in leaf angle (LANG) ( -5.97 and $-11.23 \%)$, chlorophyll content (CHLC) $(-5.48$ and $-12.15 \%)$ and all yield attributes; ear diameter (ED) $(-7.68$ and $-14.01 \%)$, number of rows per ear (NRPE) $(-6.21$ and $-9.83 \%)$, number of kernels per row (NKPR) (-7.38 and $-17.77 \%)$, and thousand kernel weight (TKW) $(-6.39$ and $-13.13 \%)$ at plant density of D2 and D3, respectively, as compared to D1. Conversely, high plant density (D2 and D3) caused a significant increase in grain yield per hectare (GYPH) compared with the low density (D1) by 8.48 and $11.23 \%$, respectively (Figure 1B). Similarly, D2 and D3 caused significant increases in days to $50 \%$ silking (DTS) (5.10 and 11.31\%), anthesis-silking interval (ASI) (12.87 and 39.88\%), plant height (PLHT) (3.78 and 9.75\%) and ear height (EHT) (6.64 and 12.86\%) as compared with low plant density (D1), respectively.

(A)

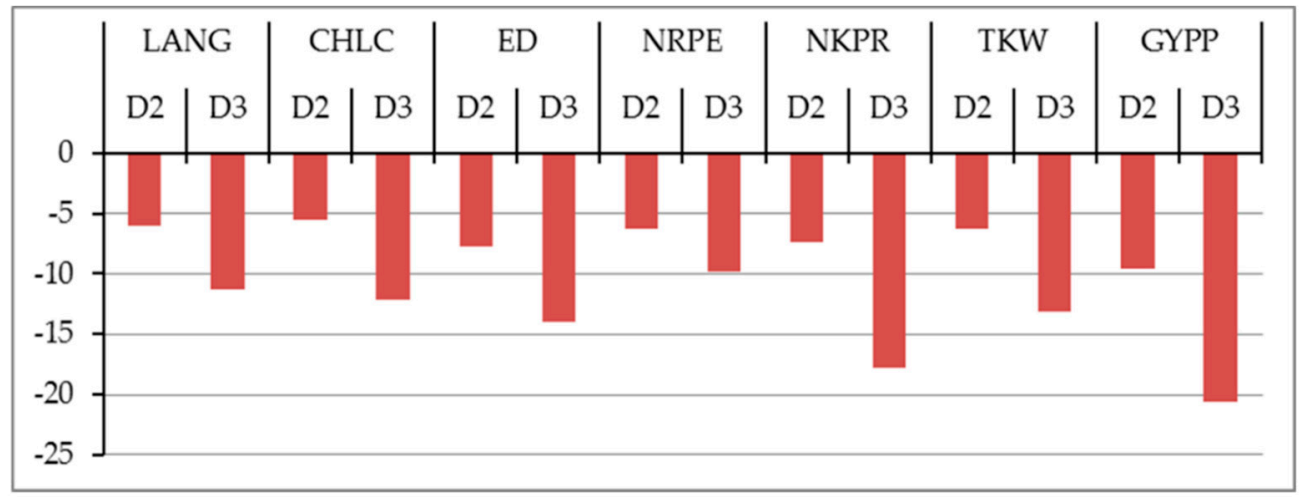

(B)

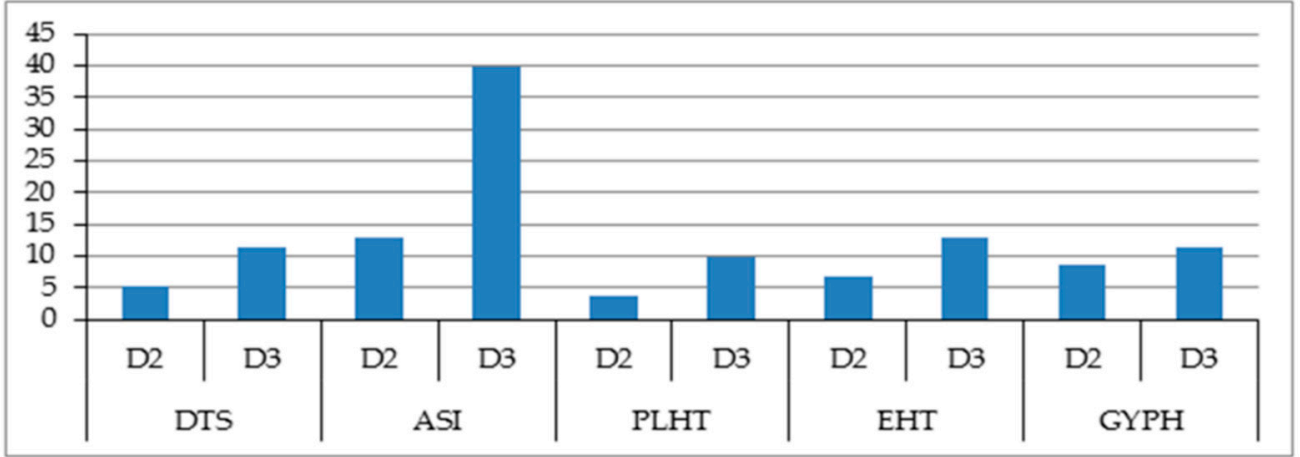

Figure 1. Shows the changes due to increased plant density: (A) reduction in leaf angle (LANG), chlorophyll content (CHLC), ear diameter (ED), number of rows per ear (NRPE), number of kernels per row (NKPR), thousand kernel weight (TKW) and grain yield per plant (GYPP); (B) increase in days to $50 \%$ silking (DTS), anthesis-silking interval (ASI), plant height (PLHT), ear height (EHT) and grain yield per hectare (GYPH) under D2 and D3 in compared with D1.

\subsection{Performance of $F_{1}$ Hybrids}

The mean performances of the $28 \mathrm{~F}_{1}$ hybrids and the commercial check hybrid SC128 for all the studied characteristics are provided in Supplementary Materials, Table S1. The evaluated hybrids showed a wide variation for all studied traits under all plant densities. The mean values for DTS were 58.22 days in $\mathrm{D} 1,61.19$ days under $\mathrm{D} 2$, and 64.80 days in $\mathrm{D} 3$ (Table 2). The earliest hybrids were $\mathrm{L}_{1} \times \mathrm{L}_{3}$ at D1, $\mathrm{L}_{3} \times \mathrm{L}_{4}$ at $\mathrm{D} 2$ and $\mathrm{L}_{1} \times \mathrm{L}_{4}$ at $\mathrm{D} 3$, while the latest hybrids were $\mathrm{L}_{6} \times \mathrm{L}_{8}$ under D1 and $\mathrm{D} 2$ and $\mathrm{L}_{3}$ $\times \mathrm{L}_{6}$ under $\mathrm{D} 3$ (Table 2). A total of 21, 17 and 4 hybrids were significantly earlier than the check hybrid SC128 under D1, D2 and D3, respectively (Supplementary Materials, Table S1). Likewise, the means of ASI were 3.26 days in D1, 3.68 days under D2, and 4.56 days in D3. The longest ASI was shown 
by the hybrid $\mathrm{L}_{3} \times \mathrm{L}_{7}$, and the shortest one was shown by $\mathrm{L}_{2} \times \mathrm{L}_{5}$ under the three plant densities (Table 2). The highest PLHT mean was $263.52 \mathrm{~cm}$ in D3, while it was $240.122 \mathrm{~cm}$ and $249.20 \mathrm{~cm}$ in D1 and D2, respectively. The tallest hybrids were $\mathrm{L}_{4} \times \mathrm{L}_{7}$ under D1 and D3, and $\mathrm{L}_{2} \times \mathrm{L}_{4}$ under D2, while the shortest hybrid was $\mathrm{L}_{2} \times \mathrm{L}_{6}$ under the three plant densities (Table 2). The means of the EHT were 117.86, 125.68 and 133.02 in D1, D2 and D3, respectively. A total of 12, 11 and 14 hybrids were significantly shorter than the check hybrid SC128 under D1, D2 and D3, respectively (Supplementary Materials, Table S1).

Table 2. Minimum, maximum and mean values of all the studied traits under three plant densities across two locations.

\begin{tabular}{|c|c|c|c|c|c|c|c|}
\hline \multirow{2}{*}{ Trait } & \multirow{2}{*}{ Parameter } & \multicolumn{2}{|c|}{ D1 } & \multicolumn{2}{|c|}{ D2 } & \multicolumn{2}{|c|}{ D3 } \\
\hline & & Value & Hybrid & Value & Hybrid & Value & Hybrid \\
\hline \multirow{3}{*}{ DTS } & Minimum & 56.17 & $\mathrm{~L}_{1} \times \mathrm{L}_{3}$ & 58.50 & $\mathrm{~L}_{3} \times \mathrm{L}_{4}$ & 62.52 & $\mathrm{~L}_{1} \times \mathrm{L}_{4}$ \\
\hline & Maximum & 61.50 & $\mathrm{~L}_{6} \times \mathrm{L}_{8}$ & 65.00 & $\mathrm{~L}_{6} \times \mathrm{L}_{8}$ & 67.60 & $\mathrm{~L}_{3} \times \mathrm{L}_{6}$ \\
\hline & Mean & \multicolumn{2}{|c|}{58.22} & \multicolumn{2}{|c|}{61.19} & \multicolumn{2}{|c|}{64.80} \\
\hline \multirow{3}{*}{ ASI } & Minimum & 2.15 & $\mathrm{~L}_{2} \times \mathrm{L}_{5}$ & 2.28 & $\mathrm{~L}_{2} \times \mathrm{L}_{5}$ & 3.12 & $\mathrm{~L}_{2} \times \mathrm{L}_{5}$ \\
\hline & Maximum & 4.65 & $\mathrm{~L}_{3} \times \mathrm{L}_{7}$ & 5.20 & $\mathrm{~L}_{3} \times \mathrm{L}_{7}$ & 5.65 & $\mathrm{~L}_{3} \times \mathrm{L}_{7}$ \\
\hline & Mean & \multicolumn{2}{|c|}{3.26} & \multicolumn{2}{|c|}{3.68} & \multicolumn{2}{|c|}{4.56} \\
\hline \multirow{3}{*}{ PLHT (cm) } & Minimum & 203.17 & $\mathrm{~L}_{2} \times \mathrm{L}_{6}$ & 206.00 & $\mathrm{~L}_{2} \times \mathrm{L}_{6}$ & 213.35 & $\mathrm{~L}_{2} \times \mathrm{L}_{6}$ \\
\hline & Maximum & 283.00 & $\mathrm{~L}_{4} \times \mathrm{L}_{7}$ & 290.63 & $\mathrm{~L}_{2} \times \mathrm{L}_{4}$ & 304.35 & $\mathrm{~L}_{4} \times \mathrm{L}_{7}$ \\
\hline & Mean & \multicolumn{2}{|c|}{240.12} & \multicolumn{2}{|c|}{249.20} & \multicolumn{2}{|c|}{263.52} \\
\hline \multirow{3}{*}{ EHT (cm) } & Minimum & 104.32 & $\mathrm{~L}_{3} \times \mathrm{L}_{6}$ & 109.49 & $\mathrm{~L}_{2} \times \mathrm{L}_{6}$ & 116.42 & $\mathrm{~L}_{2} \times \mathrm{L}_{6}$ \\
\hline & Maximum & 144.14 & $\mathrm{~L}_{6} \times \mathrm{L}_{7}$ & 151.23 & $\mathrm{~L}_{6} \times \mathrm{L}_{7}$ & 160.29 & $\mathrm{~L}_{6} \times \mathrm{L}_{7}$ \\
\hline & Mean & \multicolumn{2}{|c|}{117.86} & \multicolumn{2}{|c|}{125.68} & \multicolumn{2}{|c|}{133.02} \\
\hline \multirow{3}{*}{ LANG $\left(^{\circ}\right)$} & Minimum & 25.70 & $\mathrm{~L}_{4} \times \mathrm{L}_{5}$ & 24.40 & $\mathrm{~L}_{4} \times \mathrm{L}_{5}$ & 22.00 & $\mathrm{~L}_{4} \times \mathrm{L}_{5}$ \\
\hline & Maximum & 45.35 & $\mathrm{~L}_{3} \times \mathrm{L}_{7}$ & 42.30 & $\mathrm{~L}_{3} \times \mathrm{L}_{7}$ & 39.05 & $\mathrm{~L}_{3} \times \mathrm{L}_{7}$ \\
\hline & Mean & \multicolumn{2}{|c|}{34.03} & \multicolumn{2}{|c|}{32.00} & \multicolumn{2}{|c|}{30.21} \\
\hline \multirow{3}{*}{ CHLC (SPAD unit) } & Minimum & 44.37 & $\mathrm{~L}_{7} \times \mathrm{L}_{8}$ & 41.80 & $\mathrm{~L}_{7} \times \mathrm{L}_{8}$ & 39.39 & $\mathrm{~L}_{7} \times \mathrm{L}_{8}$ \\
\hline & Maximum & 54.50 & $\mathrm{~L}_{2} \times \mathrm{L}_{8}$ & 53.13 & $\mathrm{~L}_{2} \times \mathrm{L}_{8}$ & 50.20 & $\mathrm{~L}_{2} \times \mathrm{L}_{8}$ \\
\hline & Mean & & & & & & \\
\hline & Minimum & 4.70 & $\mathrm{~L}_{1} \times \mathrm{L}_{7}$ & 4.20 & $\mathrm{~L}_{2} \times \mathrm{L}_{4}$ & 3.60 & $\mathrm{~L}_{2} \times \mathrm{L}_{4}$ \\
\hline $\mathrm{ED}(\mathrm{cm})$ & Maximum & 5.80 & $\mathrm{~L}_{1} \times \mathrm{L}_{8}$ & 5.15 & $\mathrm{~L}_{1} \times \mathrm{L}_{3}$ & 5.00 & $\mathrm{~L}_{1} \times \mathrm{L}_{4}$ \\
\hline & Mean & & & & & & \\
\hline & Minimum & 13.00 & $\mathrm{~L}_{3} \times \mathrm{L}_{7}$ & 12.30 & $\mathrm{~L}_{3} \times \mathrm{L}_{4}$ & 12.18 & $\mathrm{~L}_{1} \times \mathrm{L}_{3}$ \\
\hline NRPE & Maximum & 16.40 & $\mathrm{~L}_{2} \times \mathrm{L}_{5}$ & 15.20 & $\mathrm{~L}_{1} \times \mathrm{L}_{5}$ & 14.70 & $\mathrm{~L}_{1} \times \mathrm{L}_{5}$ \\
\hline & Mean & & & & & & \\
\hline & Minimum & 35.20 & $\mathrm{~L}_{1} \times \mathrm{L}_{5}$ & 34.00 & $\mathrm{~L}_{1} \times \mathrm{L}_{5}$ & 30.29 & $\mathrm{~L}_{1} \times \mathrm{L}_{5}$ \\
\hline NKPR & Maximum & 45.10 & $\mathrm{~L}_{2} \times \mathrm{L}_{8}$ & 42.00 & $\mathrm{~L}_{2} \times \mathrm{L}_{8}$ & 37.95 & $\mathrm{~L}_{2} \times \mathrm{L}_{8}$ \\
\hline & Mean & & & & & & \\
\hline & Minimum & 315.00 & $\mathrm{~L}_{3} \times \mathrm{L}_{8}$ & 291.00 & $\mathrm{~L}_{5} \times \mathrm{L}_{6}$ & 276.00 & $\mathrm{~L}_{5} \times \mathrm{L}_{7}$ \\
\hline TKW (g) & Maximum & 405.00 & $\mathrm{~L}_{2} \times \mathrm{L}_{8}$ & 374.00 & $\mathrm{~L}_{1} \times \mathrm{L}_{4}$ & 353.50 & $\mathrm{~L}_{1} \times \mathrm{L}_{4}$ \\
\hline & Mean & & .00 & & & & \\
\hline & Minimum & 130.88 & $\mathrm{~L}_{3} \times \mathrm{L}_{7}$ & 122.71 & $\mathrm{~L}_{3} \times \mathrm{L}_{8}$ & 103.75 & $\mathrm{~L}_{3} \times \mathrm{L}_{7}$ \\
\hline GYPP (g) & Maximum & 236.45 & $\mathrm{~L}_{2} \times \mathrm{L}_{8}$ & 215.01 & $\mathrm{~L}_{2} \times \mathrm{L}_{8}$ & 187.44 & $\mathrm{~L}_{2} \times \mathrm{L}_{8}$ \\
\hline & Mean & & .11 & & & & \\
\hline & Minimum & 7.79 & $\mathrm{~L}_{3} \times \mathrm{L}_{7}$ & 8.76 & $\mathrm{~L}_{3} \times \mathrm{L}_{8}$ & 8.78 & $\mathrm{~L}_{3} \times \mathrm{L}_{7}$ \\
\hline GYPH $\left(\mathrm{t} \mathrm{ha}^{-1}\right)$ & Maximum & 14.07 & $\mathrm{~L}_{2} \times \mathrm{L}_{8}$ & 15.35 & $\mathrm{~L}_{2} \times \mathrm{L}_{8}$ & 15.61 & $\mathrm{~L}_{2} \times \mathrm{L}_{8}$ \\
\hline & Mean & & & & & & \\
\hline
\end{tabular}

The hybrid $\mathrm{L}_{6} \times \mathrm{L}_{7}$ had the highest ear height under the three plant densities, while the hybrids $\mathrm{L}_{3} \times \mathrm{L}_{6}$ in D1 and $\mathrm{L}_{2} \times \mathrm{L}_{6}$ under D2 and D3 had the lowest ear heights (Table 2). A total of 13, 20 and 19 hybrids had significantly lower ear placement than the check hybrid SC128 under D1, D2 and 
D3, respectively (Supplementary Materials, Table S1). Furthermore, the hybrid $\mathrm{L}_{4} \times \mathrm{L}_{5}$ displayed the lowest $\mathrm{LANG}$, while $\mathrm{L}_{3} \times \mathrm{L}_{7}$ gave the highest one under the three plant densities. The means of CHLC were 50.34, 47.59 and 44.23 SPAD units under D1, D2 and D3, respectively. The highest hybrid in CHLC was $\mathrm{L}_{2} \times \mathrm{L}_{8}$, while the lowest hybrid was $\mathrm{L}_{7} \times \mathrm{L}_{8}$ across the three plant densities (Table 2). Moreover, the hybrids $\mathrm{L}_{5} \times \mathrm{L}_{6}$ at $\mathrm{D} 1, \mathrm{~L}_{3} \times \mathrm{L}_{4}$ at $\mathrm{D} 2$ and $\mathrm{L}_{1} \times \mathrm{L}_{5}$ at $\mathrm{D} 3$ significantly surpassed the check hybrid SC128 for this trait (Table S1). The means of ED were $5.16 \mathrm{~cm}$ in D1, $4.76 \mathrm{~cm}$ under D2, and 4.44 $\mathrm{cm}$ in D3. The hybrid $\mathrm{L}_{1} \times \mathrm{L}_{7}$ at D1 and $\mathrm{L}_{2} \times \mathrm{L}_{4}$ at D2 and D3 exhibited the lowest ED, while $\mathrm{L}_{1} \times \mathrm{L}_{8}$, $\mathrm{L}_{1} \times \mathrm{L}_{3}$ and $\mathrm{L}_{1} \times \mathrm{L}_{4}$ gave the highest ones under D1, D2 and D3, respectively (Table 2). The mean for the NRPE was 14.83 in D1 and 13.91 in D2, while it was 13.37 in D3. The hybrid $\mathrm{L}_{2} \times \mathrm{L}_{5}$ under D1 and $\mathrm{L}_{1} \times \mathrm{L}_{5}$ under D2 and D3 exhibited the highest NRPE, while $\mathrm{L}_{3} \times \mathrm{L}_{7}$ in D1, $\mathrm{L}_{3} \times \mathrm{L}_{4}$ under D2 and $\mathrm{L}_{1}$ $\times \mathrm{L}_{3}$ in D3 had the lowest mean values (Table 2). Additionally, two hybrids under D1, four hybrids at D2 and three hybrids at D3 possessed higher NRPE than the check hybrid SC128 (Supplementary Materials, Table S1). The mean values of the NKPR were 40.28, 37.31 and 33.12 for D1, D2 and D3, respectively. The hybrid $\mathrm{L}_{2} \times \mathrm{L}_{8}$ had the highest NKPR, but the hybrid $\mathrm{L}_{1} \times \mathrm{L}_{5}$ displayed the lowest one under the three plant densities. Means of the TKW were $356.0 \mathrm{~g}, 333.24 \mathrm{~g}$, and $309.26 \mathrm{~g}$ in D1, $\mathrm{D} 2$, and D3, respectively. The heaviest TKW was assigned for the hybrids $\mathrm{L}_{2} \times \mathrm{L}_{8}$ under $\mathrm{D} 1$ and $\mathrm{L}_{1}$ $\times \mathrm{L}_{4}$ under D2 and D3, whereas the hybrids $\mathrm{L}_{3} \times \mathrm{L}_{8}$ in D1, $\mathrm{L}_{5} \times \mathrm{L}_{6}$ under D2 and $\mathrm{L}_{5} \times \mathrm{L}_{7}$ under D3 exhibited the lightest TKW (Table 2). Furthermore, four hybrids under D1, five hybrids at D2 and three hybrids at D3 significantly exceeded the check hybrid SC128 for this trait (Supplementary Materials, Table S1). The highest mean of GYPP was $170.11 \mathrm{~g}$ in D1, while it was 153.78 and $135.09 \mathrm{~g}$ in D2 and D3, respectively. Conversely, the highest mean of GYPH was obtained in D3 $\left(11.26 \mathrm{t} \mathrm{ha}^{-1}\right)$, followed by D2 $\left(10.98 \mathrm{t} \mathrm{ha}^{-1}\right)$ and then by D3 (10.12 $\mathrm{tha}^{-1}$ ) (Table 2). The hybrid $\mathrm{L}_{2} \times \mathrm{L}_{8}$ was the top yielding hybrid and significantly out-yielded the check hybrid SC128 by $9.98,13.16$ and $10.26 \%$ under D1, D2 and D3, respectively. Moreover, the hybrid $\mathrm{L}_{2} \times \mathrm{L}_{5}$ significantly surpassed the check hybrid SC128 by $5.26 \%$ only under D2 (Supplementary Materials, Table S1). The optimum plant density for obtaining the highest GYPH was $\mathrm{D} 3$ for all hybrids, except the hybrids; $\mathrm{L}_{2} \times \mathrm{L}_{7}, \mathrm{~L}_{3} \times \mathrm{L}_{4}, \mathrm{~L}_{3} \times \mathrm{L}_{7}$ and $\mathrm{L}_{2} \times \mathrm{L}_{8}$, where the optimum density was D2 (Supplementary Materials, Table S1). This indicates that the optimum plant density is genotype dependent and should be identified separately for each hybrid.

\subsection{General Combining Ability (GCA) Effects}

Estimates of GCA effects are presented in Table 3. High positive values of GCA effects would be of interest for all studied characteristics in question, except DTS, ASI, PLHT, EHT and LANG where high negative values would be desirable from the breeder point of view. Results showed that the highest significant and negative GCA effects under the three plant densities were obtained by the inbred lines $\mathrm{L}_{1}$ and $\mathrm{L}_{3}$ for DTS; $\mathrm{L}_{1}, \mathrm{~L}_{2}$ and $\mathrm{L}_{5}$ for ASI; $\mathrm{L}_{1}, \mathrm{~L}_{5}, \mathrm{~L}_{6}$ and $\mathrm{L}_{8}$ for PLHT; $\mathrm{L}_{3}, \mathrm{~L}_{5}$ and $\mathrm{L}_{8}$ for EHT and $\mathrm{L}_{1}, \mathrm{~L}_{2}$ and $\mathrm{L}_{4}$ for LANG. Additionally, the inbred lines $\mathrm{L}_{4}$ in D1 and D2, as well as $\mathrm{L}_{5}$ in D3 for DTS; $\mathrm{L}_{4}$ in D3 and $\mathrm{L}_{8}$ in D1 and D2 for ASI; $\mathrm{L}_{2}$ in D3 and $\mathrm{L}_{3}$ under D1 and D3 for PLHT; and $\mathrm{L}_{5}$ under D1 and D3 for LANG also expressed significant and negative GCA effects for these traits. In contrast, the inbred lines $\mathrm{L}_{1}$ in $\mathrm{D} 2$ and $\mathrm{D} 3, \mathrm{~L}_{5}$ under $\mathrm{D} 1$ and $\mathrm{L}_{2}$ under the three plant densities possessed significant and positive GCA effects for CHLC. Regarding ED, the inbred lines $\mathrm{L}_{1}$ and $\mathrm{L}_{8}$ in D1 and D3 as well as $\mathrm{L}_{3}$ in D2 had significant and positive GCA effects.

The highest positive and significant GCA effects for NRPE belonged to $\mathrm{L}_{1}$ in D2 and D3, $\mathrm{L}_{5}$ and $\mathrm{L}_{8}$ in D1 and D3, and $\mathrm{L}_{2}$ under the three plant densities. Likewise, the inbreds $\mathrm{L}_{3}$ and $\mathrm{L}_{7}$ in $\mathrm{D} 1 ; \mathrm{L}_{1}$ and $\mathrm{L}_{6}$ in D3 and L2 under the three plant densities were determined and considered to be good general combiners for NKPR. The highest positive and significant GCA effects for TKW belonged to $L_{1}$ and $L_{2}$ under the three plant densities, $\mathrm{L}_{4}$ under D1 and D2 and $\mathrm{L}_{6}$ under D3. Furthermore, the inbred lines $\mathrm{L}_{1}, \mathrm{~L}_{2}$ and $\mathrm{L}_{5}$ under the three plant densities and $\mathrm{L}_{8}$ under D3 had significant and positive GCA effects for GYPP and GYPH. Based on the summarized results, it can be concluded that parental lines $\mathrm{L}_{1}, \mathrm{~L}_{2}$ and $L_{5}$ had the highest GCA effects for grain yield and the majority of studied traits. 
Table 3. General combining ability (GCA) effects of the eight parental inbred lines for all the studied traits under three plant densities across two locations.

\begin{tabular}{|c|c|c|c|c|c|c|c|c|c|c|c|c|}
\hline \multirow{2}{*}{ Inbred Line } & \multicolumn{3}{|c|}{ DTS } & \multicolumn{3}{|c|}{ ASI } & \multicolumn{3}{|c|}{ PLHT } & \multicolumn{3}{|c|}{ EHT } \\
\hline & D1 & D2 & D3 & D1 & D2 & D3 & D1 & D2 & D3 & D1 & D2 & D3 \\
\hline $\mathrm{L}_{1}$ & $-0.84^{* *}$ & $-0.94^{* *}$ & -1.08 ** & $-0.27^{* *}$ & $-0.26^{* *}$ & $-0.16^{* *}$ & $-7.22 * *$ & $-10.48^{* *}$ & $-3.92 * *$ & $10.29^{* *}$ & $8.20^{* *}$ & $5.43^{* *}$ \\
\hline $\mathrm{L}_{2}$ & -0.21 & -0.27 & 0.07 & $-0.28^{* *}$ & $-0.31 * *$ & $-0.30 * *$ & -0.90 & -2.58 & $-7.66 * *$ & -1.35 & -1.28 & -1.54 \\
\hline $\mathrm{L}_{3}$ & $-0.62 * *$ & $-0.81^{* *}$ & $-0.38^{* *}$ & $0.43^{* *}$ & $0.48^{* *}$ & $0.37^{* *}$ & $-3.52 *$ & -1.12 & $-4.93 * *$ & $-3.74 * *$ & $-4.22 * *$ & $-3.03 * *$ \\
\hline $\mathrm{L}_{4}$ & $-0.74 * *$ & $-0.60^{* *}$ & -0.20 & -0.05 & -0.09 & $-0.23 * *$ & $18.43^{* *}$ & $24.20 * *$ & $22.27 * *$ & 1.48 & $3.60 * *$ & $4.25 * *$ \\
\hline $\mathrm{L}_{5}$ & $0.62^{* *}$ & $0.34^{*}$ & -0.56 ** & $-0.31^{* *}$ & $-0.43^{* *}$ & $-0.30^{* *}$ & $-4.29^{* *}$ & $-3.48^{*}$ & $-2.93 *$ & $-4.21^{* *}$ & $-6.60 * *$ & $-6.32 * *$ \\
\hline $\mathrm{L}_{6}$ & $1.28^{* *}$ & $1.49^{* *}$ & $1.32 * *$ & $0.65^{* *}$ & $0.58^{* *}$ & $0.42 * *$ & $-8.60 * *$ & $-9.06 * *$ & $-5.94 * *$ & 1.38 & 1.93 & $3.43 * *$ \\
\hline $\mathrm{L}_{7}$ & 0.09 & $0.30 *$ & $0.95^{* *}$ & 0.02 & 0.14 * & $0.13 *$ & $10.17^{* *}$ & $8.45^{* *}$ & $9.75^{* *}$ & $3.13^{* *}$ & $3.12 * *$ & 1.33 \\
\hline $\mathrm{L}_{8}$ & $0.43^{* *}$ & $0.49^{* *}$ & -0.12 & $-0.19 * *$ & $-0.11 *$ & 0.07 & $-4.05^{* *}$ & $-5.94 * *$ & $-6.64^{* *}$ & $-6.99 * *$ & $-4.75^{* *}$ & $-3.55^{* *}$ \\
\hline LSD 0.05 & & 0.28 & & & 0.11 & & & 2.82 & & & 2.00 & \\
\hline LSD 0.01 & & 0.36 & & & 0.15 & & & 3.70 & & & 2.63 & \\
\hline \multirow{2}{*}{ Inbred Line } & \multicolumn{3}{|c|}{ LANG } & \multicolumn{3}{|c|}{ CHLC } & \multicolumn{3}{|c|}{ ED } & \multicolumn{3}{|c|}{ NRPE } \\
\hline & D1 & D2 & D3 & D1 & D2 & D3 & D1 & D2 & D3 & D1 & D2 & D3 \\
\hline $\mathrm{L}_{1}$ & $-2.31 * *$ & $-2.55^{* *}$ & $-3.35 * *$ & 0.07 & $0.80^{* *}$ & $2.13^{* *}$ & $0.13^{*}$ & 0.08 & $0.20^{* *}$ & -0.14 & $0.33^{* *}$ & $0.45^{* *}$ \\
\hline $\mathrm{L}_{2}$ & $-1.81^{* *}$ & $-1.62^{* *}$ & $-0.92 * *$ & $1.72 * *$ & $2.58^{* *}$ & $3.18^{* *}$ & 0.11 & -0.08 & $-0.19 * *$ & $0.39 * *$ & $0.43 * *$ & $0.50 * *$ \\
\hline $\mathrm{L}_{3}$ & $5.51^{* *}$ & $5.71^{* *}$ & $5.63 * *$ & $-1.13^{* *}$ & $-1.32 * *$ & $-0.74 * *$ & -0.02 & $0.13 *$ & 0.06 & $-0.73 * *$ & $-0.55 * *$ & $-0.51^{* *}$ \\
\hline $\mathrm{L}_{4}$ & $-3.77 * *$ & $-2.92 * *$ & $-2.87^{* *}$ & -0.20 & -0.42 & $-1.20 * *$ & 0.04 & -0.03 & -0.06 & 0.13 & $-0.29 * *$ & $-0.19 *$ \\
\hline $\mathrm{L}_{5}$ & $-0.62 *$ & -0.13 & $-0.75^{* *}$ & $1.19 * *$ & -0.38 & 0.16 & -0.09 & 0.02 & -0.02 & $0.42^{* *}$ & $0.27^{* *}$ & 0.16 \\
\hline $\mathrm{L}_{6}$ & $0.84^{* *}$ & $0.55^{*}$ & $1.34^{* *}$ & $-0.44^{*}$ & $-0.61 * *$ & $-1.81^{* *}$ & $-0.21^{* *}$ & -0.11 & -0.06 & 0.16 & -0.07 & -0.08 \\
\hline $\mathrm{L}_{7}$ & $1.74^{* *}$ & $1.18^{* *}$ & $1.15^{* *}$ & $-0.97^{* *}$ & 0.00 & $-1.34^{* *}$ & -0.13 * & $-0.12 *$ & -0.07 & -0.72 ** & $-0.52 * *$ & $-0.37^{* *}$ \\
\hline $\mathrm{L}_{8}$ & 0.42 & -0.21 & -0.23 & -0.24 & $-0.66^{* *}$ & -0.38 & $0.17^{* *}$ & 0.09 & $0.13^{*}$ & $0.48^{* *}$ & $0.40 * *$ & 0.04 \\
\hline LSD 0.05 & & 0.50 & & & 0.43 & & & 0.12 & & & 0.19 & \\
\hline LSD 0.01 & & 0.66 & & & 0.56 & & & 0.15 & & & 0.25 & \\
\hline \multirow{2}{*}{ Inbred Line } & \multicolumn{3}{|c|}{ NKPR } & \multicolumn{3}{|c|}{ TKW } & \multicolumn{3}{|c|}{ GYPP } & \multicolumn{3}{|c|}{ GYPH } \\
\hline & D1 & D2 & D3 & D1 & D2 & D3 & D1 & D2 & D3 & D1 & D2 & D3 \\
\hline $\mathrm{L}_{1}$ & $-0.55^{*}$ & -0.14 & $0.58^{*}$ & $12.63^{* *}$ & $16.08^{* *}$ & $17.98^{* *}$ & $12.19^{* *}$ & $9.92^{* *}$ & $8.79^{* *}$ & $0.73^{* *}$ & $0.71^{* *}$ & $0.73 * *$ \\
\hline $\mathrm{L}_{2}$ & $1.47^{* *}$ & $1.06^{* *}$ & $1.15^{* *}$ & $18.79^{* *}$ & $16.50 * *$ & $10.23 * *$ & 19.20 ** & $19.23 * *$ & $15.67^{* *}$ & $1.14^{* *}$ & $1.37^{* *}$ & $1.30 * *$ \\
\hline $\mathrm{L}_{3}$ & $0.68^{* *}$ & $-0.55^{*}$ & $-1.17^{* *}$ & $-17.88^{* *}$ & $-10.58 * *$ & $-6.60 * *$ & $-16.55^{* *}$ & $-16.58^{* *}$ & $-16.95^{* *}$ & $-0.98 * *$ & $-1.18 * *$ & $-1.39 * *$ \\
\hline $\mathrm{L}_{4}$ & $-0.62 *$ & 0.08 & $-0.71^{* *}$ & $6.63^{* *}$ & $3.75^{*}$ & 1.81 & $-3.93^{* *}$ & 0.90 & -0.58 & $-0.23 * *$ & 0.06 & -0.05 \\
\hline $\mathrm{L}_{5}$ & $-1.43^{* *}$ & $-0.73^{* *}$ & $-0.48^{*}$ & 0.46 & -3.08 & $-5.10 * *$ & $6.92 * *$ & $4.29 * *$ & $4.19 * *$ & $0.41 * *$ & $0.31 * *$ & $0.34 * *$ \\
\hline $\mathrm{L}_{6}$ & -0.27 & 0.46 & $0.62 *$ & $-5.21 * *$ & -2.58 & $4.73 *$ & $-13.70 * *$ & $-10.97 * *$ & $-7.49^{* *}$ & $-0.82 * *$ & $-0.78 * *$ & $-0.63^{* *}$ \\
\hline $\mathrm{L}_{7}$ & $0.56 *$ & -0.01 & 0.05 & $-9.04 * *$ & -13.08 ** & $-13.27^{* *}$ & $-4.05^{* *}$ & $-4.52 * *$ & $-6.16 * *$ & $-0.24 * *$ & $-0.32 * *$ & $-0.50 * *$ \\
\hline $\mathrm{L}_{8}$ & 0.15 & -0.17 & -0.05 & $-6.38 * *$ & $-7.00 * *$ & $-9.77^{* *}$ & -0.08 & $-2.27 *$ & $2.53 *$ & 0.00 & $-0.16^{*}$ & $0.21 * *$ \\
\hline LSD 0.05 & & 0.48 & & & 3.68 & & & 2.07 & & & 0.15 & \\
\hline LSD 0.01 & & 0.63 & & & 4.84 & & & 2.72 & & & 0.20 & \\
\hline
\end{tabular}

${ }^{*}$ and ${ }^{* *}$ significant at 0.05 and 0.01 levels of probability, respectively. DTS: days to $50 \%$ silking, ASI: anthesis-silking interval, PLHT: plant height, EHT: ear height, LANG: leaf angle, CHLC: chlorophyll content, ED: ear diameter, NRPE: number of rows per ear, NKPR: number of kernels per row, TKW: thousand kernel weight, GYPP: grain yield per plant and GYPH: grain yield per hectare.

\subsection{Specific Combining Ability (SCA) Effects}

The estimated SCA values under the three plant densities across two locations are presented in Table 4. The hybrids that presented the highest significant and negatives SCA effects (desirable) under the three plant densities were $\mathrm{L}_{1} \times \mathrm{L}_{6}, \mathrm{~L}_{2} \times \mathrm{L}_{4}, \mathrm{~L}_{3} \times \mathrm{L}_{5}, \mathrm{~L}_{3} \times \mathrm{L}_{8}, \mathrm{~L}_{4} \times \mathrm{L}_{7}$ for DTS; $\mathrm{L}_{1} \times \mathrm{L}_{7}, \mathrm{~L}_{2} \times \mathrm{L}_{5}, \mathrm{~L}_{2} \times$ $\mathrm{L}_{7}, \mathrm{~L}_{2} \times \mathrm{L}_{8}, \mathrm{~L}_{3} \times \mathrm{L}_{4}, \mathrm{~L}_{3} \times \mathrm{L}_{6}$ and $\mathrm{L}_{4} \times \mathrm{L}_{5}$ for ASI; $\mathrm{L}_{1} \times \mathrm{L}_{4}, \mathrm{~L}_{2} \times \mathrm{L}_{6}, \mathrm{~L}_{2} \times \mathrm{L}_{7}, \mathrm{~L}_{2} \times \mathrm{L}_{8}, \mathrm{~L}_{3} \times \mathrm{L}_{4}$ and $\mathrm{L}_{3} \times$ $\mathrm{L}_{7}$ for PLHT; $\mathrm{L}_{1} \times \mathrm{L}_{7}, \mathrm{~L}_{1} \times \mathrm{L}_{8}, \mathrm{~L}_{2} \times \mathrm{L}_{6}$ and $\mathrm{L}_{3} \times \mathrm{L}_{6}$ for $\mathrm{EHT}$ and $\mathrm{L}_{1} \times \mathrm{L}_{4}, \mathrm{~L}_{1} \times \mathrm{L}_{5}, \mathrm{~L}_{1} \times \mathrm{L}_{6}, \mathrm{~L}_{1} \times \mathrm{L}_{7}, \mathrm{~L}_{2}$ $\times \mathrm{L}_{5}, \mathrm{~L}_{2} \times \mathrm{L}_{8}, \mathrm{~L}_{3} \times \mathrm{L}_{4}, \mathrm{~L}_{3} \times \mathrm{L}_{6}, \mathrm{~L}_{4} \times \mathrm{L}_{5}, \mathrm{~L}_{4} \times \mathrm{L}_{7}$ and $\mathrm{L}_{7} \times \mathrm{L}_{8}$ for LANG. On the contrary, the hybrid combinations; $\mathrm{L}_{1} \times \mathrm{L}_{7}, \mathrm{~L}_{2} \times \mathrm{L}_{8}, \mathrm{~L}_{3} \times \mathrm{L}_{4}$ and $\mathrm{L}_{5} \times \mathrm{L}_{6}$ for CHLC; $\mathrm{L}_{2} \times \mathrm{L}_{5}$ and $\mathrm{L}_{2} \times \mathrm{L}_{7}$ for $\mathrm{ED} ; \mathrm{L}_{1} \times \mathrm{L}_{5}, \mathrm{~L}_{2}$ $\times \mathrm{L}_{3}, \mathrm{~L}_{3} \times \mathrm{L}_{6}$ and $\mathrm{L}_{6} \times \mathrm{L}_{7}$ for NRPE; $\mathrm{L}_{1} \times \mathrm{L}_{6}, \mathrm{~L}_{2} \times \mathrm{L}_{8}$ and $\mathrm{L}_{6} \times \mathrm{L}_{7}$ for NKPE; $\mathrm{L}_{1} \times \mathrm{L}_{4}, \mathrm{~L}_{1} \times \mathrm{L}_{6}, \mathrm{~L}_{2} \times \mathrm{L}_{5}$, $\mathrm{L}_{2} \times \mathrm{L}_{8}, \mathrm{~L}_{3} \times \mathrm{L}_{5}, \mathrm{~L}_{4} \times \mathrm{L}_{5}, \mathrm{~L}_{6} \times \mathrm{L}_{7}$ and $\mathrm{L}_{7} \times \mathrm{L}_{8}$ for TKW and $\mathrm{L}_{1} \times \mathrm{L}_{3}, \mathrm{~L}_{1} \times \mathrm{L}_{6}, \mathrm{~L}_{2} \times \mathrm{L}_{5}, \mathrm{~L}_{2} \times \mathrm{L}_{8}, \mathrm{~L}_{3} \times \mathrm{L}_{4}$, $\mathrm{L}_{3} \times \mathrm{L}_{6}, \mathrm{~L}_{4} \times \mathrm{L}_{5}, \mathrm{~L}_{6} \times \mathrm{L}_{7}$ and $\mathrm{L}_{7} \times \mathrm{L}_{8}$ for GYPP and GYPH had the highest significant and positive SCA effects (desirable) under the three plant densities. Moreover, the hybrids $\mathrm{L}_{1} \times \mathrm{L}_{5}$ in D2 and D3, $\mathrm{L}_{4} \times \mathrm{L}_{7}$ in D1 and D2 and $\mathrm{L}_{2} \times \mathrm{L}_{4}$ and $\mathrm{L}_{5} \times \mathrm{L}_{7}$ under D3 displayed significant and positive SCA effects for GYPP and GYPH. It is notable that the crosses that showed high SCA effects for GYPP and GYPH also showed desirable SCA effects for some other traits, i.e., DTS, LANG, NKPE and TKW for the hybrid $\mathrm{L}_{1}$ $\times \mathrm{L}_{6}$; ASI, LANG and TKW for the two hybrids $\mathrm{L}_{2} \times \mathrm{L}_{5}$ and $\mathrm{L}_{4} \times \mathrm{L}_{5}$; ASI, PLHT, LANG, CHLC, NKPR and TKW for the hybrid $\mathrm{L}_{2} \times \mathrm{L}_{8}$ and PLHT, NRPE, NKPR and TKW for the hybrid $\mathrm{L}_{6} \times \mathrm{L}_{7}$. 
Table 4. Estimates of specific combining ability (SCA) effects of the $28 \mathrm{~F}_{1}$ crosses for all the studied traits under the three plant densities across two locations.

\begin{tabular}{|c|c|c|c|c|c|c|c|c|c|c|c|c|c|c|c|c|c|c|}
\hline \multirow{2}{*}{ Cross } & \multicolumn{3}{|c|}{ DTS } & \multicolumn{3}{|c|}{ ASI } & \multicolumn{3}{|c|}{$\begin{array}{l}\text { PLHT } \\
\end{array}$} & \multicolumn{3}{|c|}{ EHT } & \multicolumn{3}{|c|}{ LANG } & \multicolumn{3}{|c|}{ CHLC } \\
\hline & D1 & D2 & D3 & D1 & D2 & D3 & D1 & D2 & D3 & D1 & D2 & D3 & D1 & D2 & D3 & D1 & D2 & D3 \\
\hline $\mathrm{L}_{1} \times \mathrm{L}_{2}$ & 0.10 & 0.36 & 0.28 & 0.09 & 0.19 & $0.53 * *$ & $8.70^{* *}$ & 8.46 ** & $13.08 * *$ & $16.34 * *$ & 14.76 ** & $18.45^{* *}$ & $3.69^{* *}$ & $4.89^{* *}$ & $4.69^{* *}$ & $-1.90 * *$ & -2.68 ** & $-3.97 * *$ \\
\hline $\mathrm{L}_{1} \times \mathrm{L}_{3}$ & -0.49 & -0.42 & -0.53 & -0.15 & $0.31^{*}$ & $0.36^{* *}$ & 2.57 & 0.49 & $-7.16^{*}$ & $7.82^{* *}$ & $11.21^{* *}$ & $9.69^{* *}$ & $3.13^{* *}$ & $3.19^{* *}$ & $3.64^{* *}$ & 0.27 & $0.95 *$ & 0.44 \\
\hline $\mathrm{L}_{1} \times \mathrm{L}_{4}$ & -0.04 & -0.58 & $-1.03 * *$ & $0.34^{* *}$ & $0.32 *$ & $0.42^{* *}$ & $-40.63^{* *}$ & $-36.93^{* *}$ & $-29.11^{* *}$ & $6.74^{* *}$ & 1.02 & 1.82 & $-1.29 *$ & $-1.61 * *$ & $-1.92 * *$ & $-1.66^{* *}$ & $-3.95^{* *}$ & $-4.89 * *$ \\
\hline $\mathrm{L}_{1} \times \mathrm{L}_{5}$ & 0.96 ** & $0.68^{*}$ & $1.41^{* *}$ & 0.04 & 0.08 & -0.10 & $9.59^{* *}$ & 5.85 & $19.69 * *$ & $9.58^{* *}$ & $9.28 * *$ & $6.62^{* *}$ & $-2.74 * *$ & $-3.20 * *$ & $-3.79 * *$ & 0.45 & $1.01 *$ & 2.35 ** \\
\hline $\mathrm{L}_{1} \times \mathrm{L}_{6}$ & -1.05 ** & $-0.87 * *$ & $-1.49 * *$ & -0.10 & -0.18 & -0.05 & $6.40 *$ & $7.94 *$ & 3.30 & -3.99 & -4.10 & $-6.08 * *$ & $-3.21 * *$ & $-2.88 * *$ & $-1.87^{* *}$ & 0.38 & 0.84 & $2.02 * *$ \\
\hline $\mathrm{L}_{1} \times \mathrm{L}_{7}$ & 0.53 & $1.52^{* *}$ & 0.39 & $-0.55^{* *}$ & $-0.81^{* *}$ & $-0.89 * *$ & 23.63 ** & $21.17^{\text {** }}$ & 2.15 & $-20.97^{* *}$ & $-18.24^{* *}$ & $-14.06^{* *}$ & $-2.80^{* *}$ & $-2.98^{* *}$ & -2.32 ** & $1.84^{* *}$ & $1.75^{* *}$ & $2.58^{* *}$ \\
\hline $\mathrm{L}_{1} \times \mathrm{L}_{8}$ & -0.01 & $-0.67^{*}$ & $0.97^{* *}$ & $0.33^{* *}$ & 0.08 & $-0.27^{*}$ & $-10.26^{* *}$ & $-6.98 *$ & -1.95 & $-15.52^{* *}$ & $-13.93^{* *}$ & $-16.44^{* *}$ & $3.22 * *$ & $2.58 * *$ & $1.57^{* *}$ & 0.61 & $2.09 * *$ & $1.46^{* *}$ \\
\hline $\mathrm{L}_{2} \times \mathrm{L}_{3}$ & $1.21^{* *}$ & $2.26^{* *}$ & $1.16^{* *}$ & $0.74^{* *}$ & $0.65^{* *}$ & $0.72 * *$ & 22.50 ** & $34.10^{* *}$ & 35.82 ** & $5.48^{*}$ & 3.19 & 0.98 & 0.88 & 0.63 & -0.09 & -0.65 & $-1.03 *$ & -0.57 \\
\hline $\mathrm{L}_{2} \times \mathrm{L}_{4}$ & $-0.67^{*}$ & $-0.84 * *$ & -1.30 ** & $0.43 * *$ & $1.06^{* *}$ & 0.88 ** & 25.00 ** & $19.90 * *$ & $15.73^{* *}$ & 0.82 & 0.12 & -1.07 & $1.31 *$ & 0.26 & -0.60 & -0.91 & -0.10 & 0.39 \\
\hline $\mathrm{L}_{2} \times \mathrm{L}_{5}$ & $1.98^{* *}$ & -0.44 & -0.09 & $-0.53^{* *}$ & $-0.66^{* *}$ & $-0.83 * *$ & $-10.73^{* *}$ & $-10.14^{* *}$ & -5.97 & -3.86 & -2.44 & 2.48 & $-2.74 * *$ & $-1.93 * *$ & $-1.52 * *$ & $-0.97 *$ & $1.04^{*}$ & 0.04 \\
\hline $\mathrm{L}_{2} \times \mathrm{L}_{6}$ & $-0.68^{*}$ & 0.15 & $0.83^{* *}$ & 0.01 & -0.10 & -0.03 & $-27.25^{* *}$ & $-31.46^{* *}$ & $-36.41^{* *}$ & $-12.74^{* *}$ & $-16.48^{* *}$ & $-18.13^{* *}$ & -0.71 & $-2.44^{* *}$ & -1.03 & $1.66^{* *}$ & -1.53 ** & -0.52 \\
\hline $\mathrm{L}_{2} \times \mathrm{L}_{7}$ & -1.10 ** & $-1.15^{* *}$ & 0.50 & -0.30 * & $-0.61 * *$ & -0.53 ** & $-6.69 *$ & $-6.98 *$ & $-9.70^{* * *}$ & -4.10 & 2.13 & -0.21 & $2.50 * *$ & 2.93 ** & $3.40 * *$ & 0.04 & 0.65 & $1.38 * *$ \\
\hline $\mathrm{L}_{2} \times \mathrm{L}_{8}$ & $-0.84^{* *}$ & -0.34 & $-1.38^{* *}$ & $-0.45^{* *}$ & -0.52 ** & $-0.75^{* *}$ & $-11.53^{* *}$ & $-13.88^{* *}$ & $-12.56^{* *}$ & -1.94 & -1.28 & -2.51 & -4.93 ** & $-4.35^{* *}$ & $-4.86^{* *}$ & $2.73^{* *}$ & $3.64^{* *}$ & $3.25^{* *}$ \\
\hline $\mathrm{L}_{3} \times \mathrm{L}_{4}$ & $1.19^{* *}$ & -1.21 ** & 0.34 & $-0.89^{* *}$ & $-1.30^{* *}$ & $-1.31 * *$ & $-15.53^{* *}$ & $-15.19^{* *}$ & $-12.24^{* *}$ & 3.66 & 3.71 & -1.88 & $-4.06^{* *}$ & $-3.97^{* *}$ & $-2.44^{* *}$ & $3.44^{* *}$ & $5.64^{* *}$ & $6.31^{* *}$ \\
\hline $\mathrm{L}_{3} \times \mathrm{L}_{5}$ & -1.62 ** & $-0.65^{*}$ & $-1.04 * *$ & $0.61^{* *}$ & $0.77^{* *}$ & $0.62 * *$ & 5.89 & 3.14 & 0.11 & -3.62 & 1.51 & -0.31 & $1.89^{* *}$ & $1.74^{* *}$ & $1.24^{*}$ & $-3.44 * *$ & $-2.37^{* *}$ & $-3.38^{* *}$ \\
\hline $\mathrm{L}_{3} \times \mathrm{L}_{6}$ & -0.27 & $-1.05^{* *}$ & $1.82^{* *}$ & $-0.87^{\text {** }}$ & $-0.97^{\text {** }}$ & $-0.77^{* *}$ & 5.30 & -4.02 & 0.10 & $-11.04^{* *}$ & -10.35 ** & $-7.78^{* *}$ & $-5.82 * *$ & -3.94 ** & -3.81 ** & $-1.65^{* *}$ & $-3.64^{* *}$ & $-1.92^{* *}$ \\
\hline $\mathrm{L}_{3} \times \mathrm{L}_{7}$ & $1.41^{* *}$ & $1.89 * *$ & -0.01 & $0.93^{* *}$ & $0.90 * *$ & $0.59 * *$ & $-18.37^{* * *}$ & $-12.94^{* *}$ & $-13.61^{* *}$ & -0.76 & $-5.93^{* *}$ & $-7.95 * *$ & $4.18^{* *}$ & $3.53 * *$ & $2.14^{* *}$ & $2.44^{* *}$ & $2.35^{* *}$ & 0.92 \\
\hline $\mathrm{L}_{3} \times \mathrm{L}_{8}$ & $-1.43^{* *}$ & $-0.80^{* *}$ & $-1.74^{* *}$ & $-0.37^{* *}$ & $-0.36^{* *}$ & -0.22 & -2.36 & -5.59 & -3.01 & -1.55 & -3.34 & $7.25^{* *}$ & -0.20 & $-1.18^{*}$ & -0.67 & -0.42 & $-1.89 * *$ & $-1.81^{* *}$ \\
\hline $\mathrm{L}_{4} \times \mathrm{L}_{5}$ & -1.10 ** & -0.06 & -0.17 & $-0.47^{* *}$ & $-0.36^{* *}$ & $-0.40 * *$ & -1.56 & 4.05 & $9.83^{* *}$ & -1.54 & -3.17 & 0.54 & -3.82 ** & $-4.42 * *$ & $-3.97 * *$ & 0.05 & -0.77 & $-1.00 *$ \\
\hline $\mathrm{L}_{4} \times \mathrm{L}_{6}$ & -0.16 & $1.74^{* *}$ & $0.64^{*}$ & 0.02 & $0.33^{*}$ & 0.21 & 16.55 ** & $24.75^{* *}$ & 13.42 ** & $-7.77^{* * *}$ & $-5.22 *$ & -2.11 & $6.61 * *$ & $7.69 * *$ & $6.35^{* *}$ & $-2.34 * *$ & -0.54 & $-1.15^{*}$ \\
\hline $\mathrm{L}_{4} \times \mathrm{L}_{7}$ & $-0.67^{*}$ & -0.82 ** & $-0.88^{* *}$ & $0.34^{* *}$ & 0.01 & 0.25 & $14.48^{* *}$ & $7.99 *$ & $8.97^{* *}$ & -1.93 & -0.52 & 0.89 & $-2.63^{* *}$ & $-3.24^{* *}$ & $-3.67^{* *}$ & -0.24 & -0.28 & -0.59 \\
\hline $\mathrm{L}_{4} \times \mathrm{L}_{8}$ & $1.44^{* *}$ & $1.79^{* *}$ & $2.39 * *$ & 0.23 & -0.06 & -0.04 & 1.69 & -4.58 & $-6.59 *$ & 0.03 & 4.06 & 1.81 & $3.88^{* *}$ & $5.28 * *$ & $6.25^{* *}$ & $1.66^{* *}$ & 0.01 & 0.92 \\
\hline $\mathrm{L}_{5} \times \mathrm{L}_{6}$ & 0.49 & 0.05 & $-1.49^{* *}$ & 0.03 & 0.02 & 0.11 & -5.53 & $-7.57^{*}$ & $-11.28 * *$ & -1.55 & -0.52 & 0.62 & 0.31 & 0.40 & -0.27 & $2.56^{* *}$ & $2.02 * *$ & $2.80^{* *}$ \\
\hline $\mathrm{L}_{5} \times \mathrm{L}_{7}$ & -0.03 & $1.39^{* *}$ & $0.68^{*}$ & $-0.27^{*}$ & -0.21 & -0.12 & -4.32 & -5.58 & $-16.94 * *$ & 1.50 & -0.83 & $-5.39 *$ & $3.57 * *$ & $3.94^{* *}$ & $5.21^{* *}$ & 0.09 & $-0.99 *$ & -0.47 \\
\hline $\mathrm{L}_{5} \times \mathrm{L}_{8}$ & $-0.67^{*}$ & $-0.95^{* *}$ & $0.70 *$ & $0.58^{* *}$ & 0.36 ** & 0.72 ** & $6.66^{*}$ & $10.24^{* *}$ & 4.57 & -0.51 & -3.83 & $-4.56^{*}$ & $3.53^{* *}$ & $3.46^{* *}$ & $3.10^{* *}$ & $1.26^{* *}$ & 0.07 & -0.33 \\
\hline $\mathrm{L}_{6} \times \mathrm{L}_{7}$ & 0.0 & -1.91 ** & -0.01 & $0.54^{* *}$ & $0.55^{* *}$ & $0.34^{* *}$ & $-9.99 * *$ & $-7.05^{*}$ & $20.23^{* *}$ & $21.93^{* *}$ & $20.87^{* *}$ & $22.87^{* *}$ & $1.75^{* *}$ & $1.39 *$ & 0.63 & 0.53 & $1.64 * *$ & -0.78 \\
\hline $\mathrm{L}_{6} \times \mathrm{L}_{8}$ & $1.67^{* *}$ & $1.90^{* *}$ & -0.29 & $0.37^{* *}$ & $0.34^{* *}$ & 0.19 & $14.52 * *$ & $17.40^{* *}$ & $10.65^{* *}$ & $15.17^{* *}$ & $15.80 * *$ & 10.60 ** & 1.07 & -0.22 & 0.02 & $-1.15^{*}$ & $1.20 *$ & -0.45 \\
\hline $\mathrm{L}_{7} \times \mathrm{L}_{8}$ & -0.15 & -0.91 ** & $-0.66^{*}$ & $-0.69 * *$ & 0.18 & $0.36^{* *}$ & 1.26 & 3.38 & $8.90 * *$ & 4.33 & 2.52 & 3.85 & $-6.57^{* *}$ & $-5.56^{* * *}$ & $-5.40^{* *}$ & $-4.70^{* *}$ & $-5.11^{* *}$ & $-3.04^{* *}$ \\
\hline LSD 0.05 & & 0.61 & & & 0.25 & & & 6.23 & & & 4.42 & & & 1.12 & & & 0.95 & \\
\hline LSD 0.01 & & 0.80 & & & 0.33 & & & 8.19 & & & 5.81 & & & 1.47 & & & 1.24 & \\
\hline
\end{tabular}


Table 4. Cont

\begin{tabular}{|c|c|c|c|c|c|c|c|c|c|c|c|c|c|c|c|c|c|c|}
\hline \multirow{2}{*}{ Cross } & \multicolumn{3}{|c|}{ ED } & \multicolumn{3}{|c|}{ NRPE } & \multicolumn{3}{|c|}{ NKPR } & \multicolumn{3}{|c|}{ TKW } & \multicolumn{3}{|c|}{ GYPP } & \multicolumn{3}{|c|}{ GYPH } \\
\hline & D1 & D2 & D3 & D1 & D2 & D3 & D1 & D2 & D3 & D1 & D2 & D3 & D1 & D2 & D3 & D1 & D2 & D3 \\
\hline $\mathrm{L}_{1} \times \mathrm{L}_{2}$ & -0.13 & -0.17 & -0.14 & -0.05 & -0.16 & -0.31 & -0.74 & -0.47 & $-1.83^{* *}$ & $13.26^{* * *}$ & 6.92 & 3.06 & 0.63 & -1.34 & -2.05 & 0.04 & -0.10 & -0.16 \\
\hline $\mathrm{L}_{1} \times \mathrm{L}_{3}$ & 0.21 & 0.17 & -0.19 & -0.69 ** & $-0.88^{* *}$ & -1.12 ** & -1.34 * & 0.43 & $1.38^{*}$ & 3.93 & 2.00 & -6.11 & 16.42 ** & $10.99 * *$ & $10.06^{* *}$ & $0.98^{* *}$ & $0.78 * *$ & $0.82 * *$ \\
\hline $\mathrm{L}_{1} \times \mathrm{L}_{4}$ & $0.32 *$ & 0.19 & $0.42 * *$ & 0.20 & $0.76^{* *}$ & $0.50 *$ & 0.96 & 0.97 & 0.03 & 27.43 ** & $21.67 * *$ & $24.48^{* *}$ & $-24.25 * *$ & $-22.70^{* *}$ & -18.21 ** & $-1.44^{* *}$ & $-1.62 * *$ & $-1.51 * *$ \\
\hline $\mathrm{L}_{1} \times \mathrm{L}_{5}$ & $-0.34^{* *}$ & -0.07 & 0.11 & $0.42 *$ & $0.69 * *$ & $0.73^{* *}$ & $-3.04^{* *}$ & $-2.34 * *$ & $-1.39 * *$ & $-33.40^{* *}$ & $-24.50^{* *}$ & $-16.11^{* *}$ & 2.81 & $10.32^{* *}$ & $12.43^{* *}$ & 0.17 & $0.74^{* *}$ & $1.04^{* *}$ \\
\hline $\mathrm{L}_{1} \times \mathrm{L}_{6}$ & 0.03 & 0.16 & 0.12 & -0.18 & -0.36 & -0.30 & $2.61^{* *}$ & $2.77^{* *}$ & $2.93 * *$ & $25.26 * *$ & $25.00^{* *}$ & 22.06 ** & $33.52 * *$ & $28.56^{* *}$ & $22.11 * *$ & $1.99^{* *}$ & $2.04^{* *}$ & $1.85^{* *}$ \\
\hline $\mathrm{L}_{1} \times \mathrm{L}_{7}$ & $-0.45^{* *}$ & -0.13 & $-0.26^{*}$ & 0.25 & 0.29 & 0.15 & -0.63 & $-2.06^{* *}$ & $-1.10^{*}$ & $-9.90 *$ & -10.50 * & -6.94 & $-18.98^{* *}$ & $-21.89^{* *}$ & -18.42 ** & $-1.13^{* *}$ & $-1.56^{* *}$ & $-1.55^{* *}$ \\
\hline $\mathrm{L}_{1} \times \mathrm{L}_{8}$ & $0.35^{* *}$ & -0.14 & -0.06 & 0.05 & -0.33 & 0.35 & $2.19^{* *}$ & 0.70 & -0.03 & $-26.57 * *$ & $-20.58^{* *}$ & $-20.44^{* *}$ & $-10.14^{* *}$ & -3.94 & $-5.92 *$ & -0.60 ** & -0.28 & $-0.49^{* *}$ \\
\hline $\mathrm{L}_{2} \times \mathrm{L}_{3}$ & -0.05 & 0.08 & $-0.31^{*}$ & $0.74^{* *}$ & $0.82^{* *}$ & $1.05^{* *}$ & $2.53^{* *}$ & 0.28 & -2.66 ** & $-12.24^{* *}$ & -7.42 & 1.14 & $-15.69^{* *}$ & $-15.14^{* *}$ & $-6.32 * *$ & -0.93 ** & $-1.08^{* *}$ & $-0.54^{* *}$ \\
\hline $\mathrm{L}_{2} \times \mathrm{L}_{4}$ & -0.51 ** & $-0.46^{* *}$ & $-0.59^{* *}$ & $-1.02^{* *}$ & -0.04 & 0.02 & $-2.07^{* *}$ & $-1.56^{* *}$ & -0.02 & $-12.74^{* *}$ & $-9.75 *$ & $-14.77^{* * *}$ & -4.15 & 2.88 & $8.81^{* *}$ & -0.25 & 0.21 & $0.74^{* *}$ \\
\hline $\mathrm{L}_{2} \times \mathrm{L}_{5}$ & $0.42 * *$ & $0.29 *$ & $0.57^{* *}$ & $0.79 * *$ & 0.39 & 0.18 & 0.00 & $1.13^{*}$ & 1.03 & $18.43 * *$ & $22.58^{* * *}$ & $14.64^{* *}$ & $25.96^{* *}$ & $23.99 * *$ & $18.55^{* *}$ & $1.54^{* *}$ & $1.71^{* *}$ & $1.55^{* *}$ \\
\hline $\mathrm{L}_{2} \times \mathrm{L}_{6}$ & -0.05 & -0.08 & 0.01 & $-0.73^{* *}$ & $-0.96^{* *}$ & $-1.18^{* *}$ & $-4.42^{* *}$ & $-3.73 * *$ & $-1.99 * *$ & $-18.90^{* *}$ & -14.42 ** & -6.69 & $-33.57^{* *}$ & $-30.75^{* *}$ & $-29.77^{* *}$ & -2.00 *** & $-2.20^{* *}$ & $-2.47^{* *}$ \\
\hline $\mathrm{L}_{2} \times \mathrm{L}_{7}$ & 0.36 ** & 0.43 ** & $0.63 * *$ & 0.33 & -0.31 & -0.30 & $1.45^{* *}$ & 0.44 & $1.60 * *$ & $-25.07^{* *}$ & $-25.92 * *$ & $-23.69^{* *}$ & $-21.99 * *$ & $-25.20 * *$ & $-24.60^{* *}$ & $-1.31^{* *}$ & $-1.80 * *$ & $-2.07^{* *}$ \\
\hline $\mathrm{L}_{2} \times \mathrm{L}_{8}$ & -0.04 & -0.08 & -0.17 & -0.05 & 0.27 & $0.54^{*}$ & $3.26^{* *}$ & 3.90 ** & $3.88^{* *}$ & 37.26 ** & 28.00 ** & $26.31^{* *}$ & 48.82 ** & $45.56^{* *}$ & $35.39 * *$ & $2.90^{* *}$ & $3.25 * *$ & $2.95 * *$ \\
\hline $\mathrm{L}_{3} \times \mathrm{L}_{4}$ & -0.18 & -0.06 & 0.16 & -0.41 & $-0.76^{* *}$ & $-0.47^{*}$ & 0.62 & -0.34 & $1.78^{* *}$ & $10.93^{* * *}$ & 7.33 & $-17.94 * *$ & $21.41^{* *}$ & $20.99 * *$ & $9.83 * *$ & $1.27^{* *}$ & $1.50 * *$ & $0.80 * *$ \\
\hline $\mathrm{L}_{3} \times \mathrm{L}_{5}$ & -0.05 & 0.08 & 0.12 & $-0.49^{*}$ & -0.12 & 0.19 & $2.83^{* *}$ & $2.38^{* *}$ & 0.60 & $27.10^{* * *}$ & $15.17^{* * *}$ & 22.98 ** & $-15.18^{* *}$ & $-17.28^{* *}$ & $-14.84^{* *}$ & -0.90 ** & $-1.23 * *$ & $-1.25 * *$ \\
\hline $\mathrm{L}_{3} \times \mathrm{L}_{6}$ & -0.12 & -0.09 & $0.36^{* *}$ & $1.07^{* *}$ & $0.72^{* *}$ & $0.93^{* *}$ & -0.33 & -0.91 & -1.05 & -2.24 & 4.67 & 5.14 & $16.68^{* *}$ & $10.35 * *$ & $14.54^{* *}$ & $0.99^{* *}$ & $0.74^{* *}$ & $1.20^{* *}$ \\
\hline $\mathrm{L}_{3} \times \mathrm{L}_{7}$ & -0.01 & -0.18 & -0.12 & -0.36 & -0.03 & 0.11 & $-2.86^{* *}$ & -0.35 & -0.18 & $-11.40^{* *}$ & $-10.83^{* *}$ & -0.86 & -17.02 ** & 1.01 & $-6.99 * *$ & $-1.01^{* *}$ & 0.07 & $-0.48^{* *}$ \\
\hline $\mathrm{L}_{3} \times \mathrm{L}_{8}$ & 0.19 & 0.01 & -0.02 & 0.14 & 0.25 & $-0.69^{* *}$ & $-1.44^{* *}$ & $-1.49^{* *}$ & 0.12 & $-16.07^{* *}$ & $-10.92^{* *}$ & -4.36 & $-6.61^{* *}$ & $-10.93^{* *}$ & $-6.29^{* *}$ & $-0.39 *$ & $-0.78^{* *}$ & $-0.54^{* *}$ \\
\hline $\mathrm{L}_{4} \times \mathrm{L}_{5}$ & 0.19 & 0.14 & -0.06 & -0.04 & 0.38 & 0.06 & -0.57 & -0.66 & $1.09^{*}$ & $22.60 * *$ & 29.83 ** & $38.56^{* *}$ & $31.54 * *$ & 18.23 ** & $14.79 * *$ & $1.88^{* *}$ & $1.30^{* *}$ & $1.24^{* *}$ \\
\hline $\mathrm{L}_{4} \times \mathrm{L}_{6}$ & 0.22 & 0.18 & 0.08 & 0.21 & $0.46^{*}$ & 0.11 & 0.67 & 0.15 & $-2.01^{* *}$ & 3.26 & 2.33 & $8.73^{*}$ & $-9.18^{* *}$ & $-6.02 * *$ & $-5.72 *$ & $-0.55^{* *}$ & $-0.43^{*}$ & $-0.47^{* *}$ \\
\hline $\mathrm{L}_{4} \times \mathrm{L}_{7}$ & -0.07 & -0.16 & -0.11 & $0.78^{* *}$ & 0.21 & 0.29 & $1.84^{* *}$ & $1.72 * *$ & 0.26 & $-23.90 * *$ & $-22.17^{* *}$ & $-19.27^{* *}$ & $12.90^{* *}$ & $5.74^{*}$ & 0.74 & $0.77^{* *}$ & $0.41 *$ & 0.05 \\
\hline $\mathrm{L}_{4} \times \mathrm{L}_{8}$ & 0.03 & 0.18 & 0.09 & 0.28 & $-1.01^{* *}$ & $-0.52 *$ & $-1.44^{* *}$ & -0.27 & $-1.14^{*}$ & $-27.57^{* *}$ & $-29.25 * *$ & $-19.77^{* *}$ & $-28.26^{* *}$ & $-19.12^{* *}$ & $-10.25^{* *}$ & -1.68 ** & $-1.37^{* *}$ & -0.85 ** \\
\hline $\mathrm{L}_{5} \times \mathrm{L}_{6}$ & -0.05 & -0.18 & $-0.36^{* *}$ & -0.38 & $-0.91 * *$ & -0.23 & $1.38^{*}$ & 0.87 & $1.30^{*}$ & $-32.57^{* *}$ & $-35.83^{* *}$ & $-31.86^{* *}$ & -21.29 ** & $-10.90^{* *}$ & $-12.19^{* *}$ & $-1.27^{* *}$ & $-0.78^{* *}$ & $-1.01^{* *}$ \\
\hline $\mathrm{L}_{5} \times \mathrm{L}_{7}$ & 0.06 & -0.07 & -0.14 & -0.90 ** & $-0.86^{* *}$ & $-0.91 * *$ & $-1.65^{* * *}$ & $-1.07 *$ & $-2.07 * *$ & -2.74 & -5.33 & -14.36 ** & -4.05 & 0.14 & $4.58^{*}$ & -0.24 & 0.01 & 0.36 * \\
\hline
\end{tabular}


Table 4. Cont.

\begin{tabular}{|c|c|c|c|c|c|c|c|c|c|c|c|c|c|c|c|c|c|c|}
\hline \multirow{2}{*}{ Cross } & \multicolumn{3}{|c|}{ ED } & \multicolumn{3}{|c|}{ NRPE } & \multicolumn{3}{|c|}{ NKPR } & \multicolumn{3}{|c|}{ TKW } & \multicolumn{3}{|c|}{ GYPP } & \multicolumn{3}{|c|}{ GYPH } \\
\hline & D1 & D2 & D3 & D1 & D2 & D3 & D1 & D2 & D3 & D1 & D2 & D3 & D1 & D2 & D3 & D1 & D2 & D3 \\
\hline $\mathrm{L}_{5} \times \mathrm{L}_{8}$ & -0.24 & -0.18 & -0.24 & $0.60^{* *}$ & $0.43^{*}$ & -0.02 & $1.06^{*}$ & -0.31 & -0.57 & 0.60 & -1.92 & $-13.86^{* *}$ & $-19.79 * *$ & $-24.51^{* *}$ & $-23.32^{* *}$ & $-1.18^{* *}$ & $-1.75^{* *}$ & $-1.94^{* *}$ \\
\hline $\mathrm{L}_{6} \times \mathrm{L}_{7}$ & 0.19 & -0.04 & -0.31 * & $0.46^{*}$ & $0.69^{* *}$ & 0.49 * & $2.79 * *$ & $2.35^{* *}$ & $2.28^{* *}$ & $32.93^{* * *}$ & $29.17^{* *}$ & $17.81^{* *}$ & $23.51^{* *}$ & $18.01^{* *}$ & $22.66^{* *}$ & $1.40^{* *}$ & $1.29^{* *}$ & $1.87^{* *}$ \\
\hline $\mathrm{L}_{6} \times \mathrm{L}_{8}$ & -0.21 & 0.05 & 0.09 & $-0.45^{*}$ & 0.37 & 0.18 & $-2.69 * *$ & $-1.50 * *$ & $-1.47^{* *}$ & -7.74 & $-10.92 * *$ & $-15.19^{* *}$ & $-9.67 * *$ & $-9.25 * *$ & $-11.64^{* *}$ & $-0.58^{* *}$ & $-0.66^{* *}$ & $-0.96^{* *}$ \\
\hline $\mathrm{L}_{7} \times \mathrm{L}_{8}$ & -0.09 & 0.16 & 0.31 * & $-0.57^{* * *}$ & 0.02 & 0.16 & -0.93 & -1.03 & -0.79 & $40.10 * *$ & $45.58^{* * *}$ & $47.31 * *$ & $25.64 * *$ & $22.19 * *$ & $22.03 * *$ & $1.53^{* *}$ & $1.58^{* *}$ & 1.82 ** \\
\hline LSD 0.05 & & 0.26 & & & 0.42 & & & 1.06 & & & 8.15 & & & 4.57 & & & 0.34 & \\
\hline LSD 0.01 & & 0.34 & & & 0.55 & & & 1.39 & & & 10.71 & & & 6.01 & & & 0.45 & \\
\hline
\end{tabular}

* and ** significant at 0.05 and 0.01 levels of probability, respectively. DTS: days to 50\% silking, ASI: anthesis-silking interval, PLHT: plant height, EHT: ear height, LANG: leaf angle, CHLC: chlorophyll content, ED: ear diameter, NRPE: number of rows per ear, NKPR: number of kernels per row, TKW: thousand kernel weight, GYPP: grain yield per plant and GYPH: grain yield per hectare. 


\subsection{SSR Polymorphisms, Genetic Distance (GD) and Cluster Analysis}

Out of twenty-two SSR primer pairs analyzed, ten were polymorphic among the eight inbreds studied (Table 5). The primer pairs generated a total of 80 polymorphic fragments (Figure 2). The number of alleles per locus ranged from 2 to 6 , with an average number of 2.7 alleles/locus (Table 5). The major allele frequency had an average of 0.59 with a range extended from 0.25 to 0.88 . The gene diversity and polymorphic information content (PIC) averaged 0.50 and 0.41 , with ranges of $0.22-0.81$ and $0.19-0.79$, respectively. The umc1033 locus showed the highest gene diversity and PIC (Table 5). Genetic distance estimates based on SSR markers ranged from 0.31 to 0.78 with an average of 0.61 (Table 6). The lowest genetic distance (0.31) was obtained between the inbred lines $\left(\mathrm{L}_{1}\right.$ and $\left.\mathrm{L}_{4}\right)$, whereas the highest genetic distance (0.78) was observed between the inbred lines $\left(\mathrm{L}_{1}\right.$ and $\left.\mathrm{L}_{8}\right),\left(\mathrm{L}_{2}\right.$ and $\left.\mathrm{L}_{5}\right),\left(\mathrm{L}_{2}\right.$ and $\left.\mathrm{L}_{6}\right)$ and $\left(\mathrm{L}_{2}\right.$ and $\left.\mathrm{L}_{8}\right)$. The dendrogram constructed based on GD revealed two main clusters; $\mathrm{L}_{1}, \mathrm{~L}_{2}$, $\mathrm{L}_{3}$ and $\mathrm{L}_{4}$ constituted the first group, while $\mathrm{L}_{5}, \mathrm{~L}_{6}, \mathrm{~L}_{7}$ and $\mathrm{L}_{8}$ formed the second one (Figure 3).

Table 5. Number of alleles, major allele frequency, gene diversity and polymorphic information content (PIC) of the ten SSR markers used in this study.

\begin{tabular}{ccccccc}
\hline Marker & Ch. & Size Range (bp) & No. of Alleles & Major Allele Frequency & Gene Diversity & PIC \\
\hline phi308707 & 1 & $125-140$ & 2 & 0.63 & 0.47 & 0.36 \\
phi96100 & 2 & $150-200$ & 2 & 0.88 & 0.22 & 0.19 \\
phi453121 & 3 & $150-200$ & 2 & 0.50 & 0.50 & 0.38 \\
phi072 & 4 & $100-150$ & 2 & 0.75 & 0.38 & 0.30 \\
phi024 & 5 & $100-200$ & 2 & 0.50 & 0.50 & 0.38 \\
umc1014 & 6 & $100-150$ & 3 & 0.50 & 0.59 & 0.51 \\
phi112 & 7 & $150-200$ & 3 & 0.50 & 0.59 & 0.51 \\
phi015 & 8 & $50-150$ & 3 & 0.50 & 0.81 & 0.79 \\
umc1033 & 9 & $50-200$ & 6 & 0.25 & 0.22 & 0.19 \\
phi301654 & 10 & $100-150$ & 2 & 0.88 & 0.50 & 0.41 \\
\hline
\end{tabular}
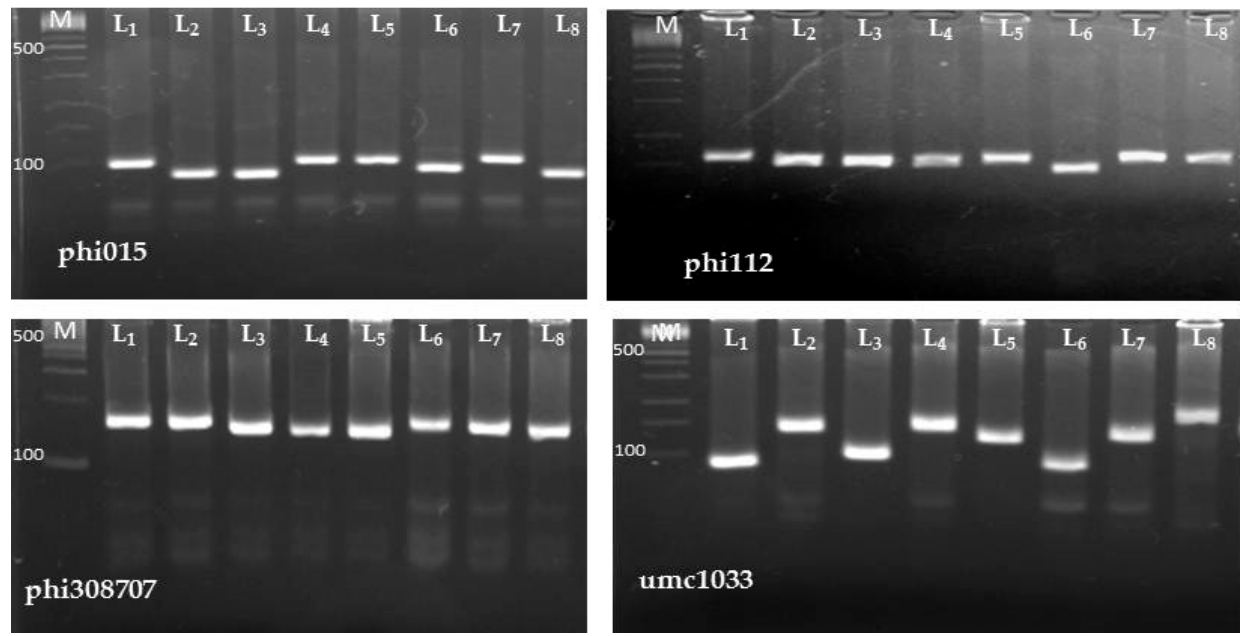

Figure 2. Amplification pattern of representative SSR markers with the eight maize inbred lines $\left(\mathrm{L}_{1}-\mathrm{L}_{8}\right)$. $\mathrm{M}$ refers to the $100 \mathrm{bp}$ DNA ladder. 
Table 6. Genetic distance (GD) matrix among the eight maize inbred lines based on SSR analysis.

\begin{tabular}{ccccccccc}
\hline Parent & $\mathbf{L}_{\mathbf{1}}$ & $\mathbf{L}_{\mathbf{2}}$ & $\mathbf{L}_{\mathbf{3}}$ & $\mathbf{L}_{\mathbf{4}}$ & $\mathbf{L}_{\mathbf{5}}$ & $\mathbf{L}_{\mathbf{6}}$ & $\mathbf{L}_{\mathbf{7}}$ & $\mathbf{L}_{\mathbf{8}}$ \\
\hline $\mathrm{L}_{1}$ & - & 0.43 & 0.53 & 0.31 & 0.71 & 0.71 & 0.71 & 0.78 \\
$\mathrm{~L}_{2}$ & & - & 0.43 & 0.53 & 0.78 & 0.78 & 0.71 & 0.78 \\
$\mathrm{~L}_{3}$ & & & - & 0.43 & 0.63 & 0.63 & 0.63 & 0.71 \\
$\mathrm{~L}_{4}$ & & & & - & 0.63 & 0.71 & 0.63 & 0.71 \\
$\mathrm{~L}_{5}$ & & & & & - & 0.63 & 0.43 & 0.71 \\
$\mathrm{~L}_{6}$ & & & & & & - & 0.43 & 0.63 \\
$\mathrm{~L}_{7}$ & & & & & & & - & 0.53 \\
$\mathrm{~L}_{8}$ & & & & & & & & - \\
\hline
\end{tabular}

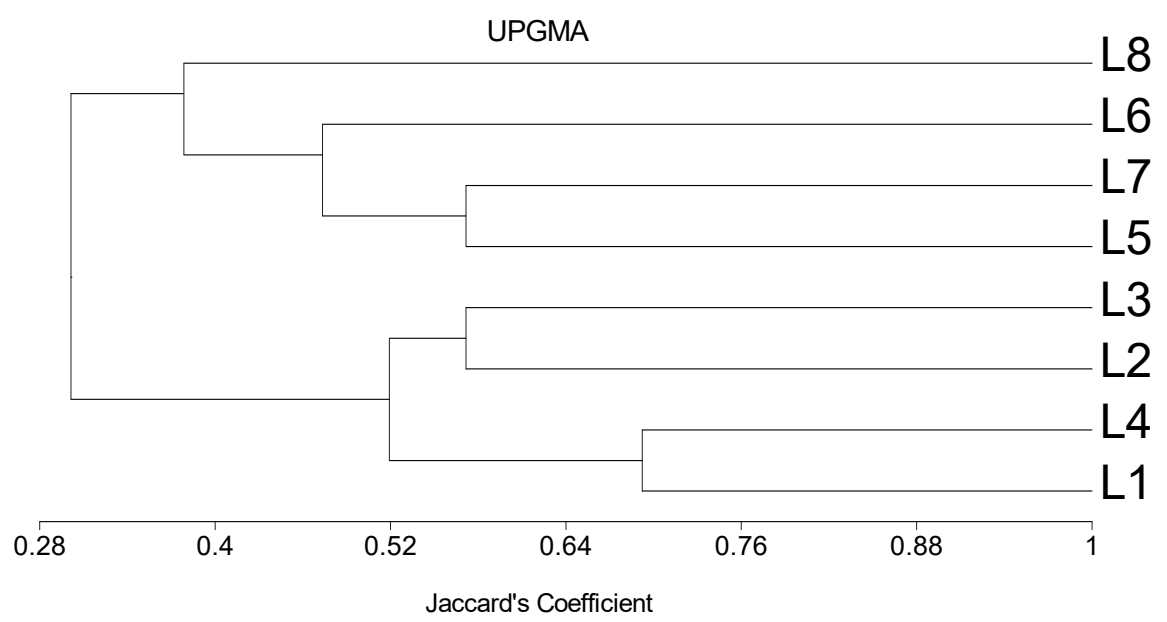

Figure 3. Dendrogram of the eight maize inbred lines constructed from SSR data using (UPGMA) according to Jaccard's coefficients.

\subsection{Association between Genetic Distance, $F_{1}$ Hybrid Performance and SCA}

Correlations between GD estimated for pairs of inbred lines with each of $\mathrm{F}_{1}$ hybrid performance and SCA were not significant for all measured traits (Table 7, Figure 4A,B). However, significant and positive association was observed between $\mathrm{F}_{1}$ hybrid performance and SCA for all the studied traits across the three plant densities (Table 7).

Table 7. Correlation coefficients among parental genetic distance (GD), $\mathrm{F}_{1}$ hybrid performance and SCA for all studied traits across all environments.

\begin{tabular}{|c|c|c|c|c|c|c|c|c|c|c|c|c|}
\hline Trait & DTS & ASI & PLHT & EHT & LANG & CHLC & ED & NRPE & NKPR & TKW & GYPP & GYPH \\
\hline $\mathrm{r}\left(\mathrm{GD}, \mathrm{F}_{1}\right)$ & 0.20 & -0.26 & -0.20 & -0.60 & -0.09 & 0.30 & 0.13 & 0.26 & 0.04 & -0.21 & 0.05 & 0.05 \\
\hline $\mathrm{r}(\mathrm{GD}, \mathrm{SCA})$ & 0.01 & -0.26 & 0.00 & -0.55 & -0.07 & 0.29 & 0.11 & 0.12 & -0.25 & -0.26 & 0.04 & 0.04 \\
\hline $\mathrm{r}\left(\mathrm{F}_{1}, \mathrm{SCA}\right)$ & $0.69 * *$ & $0.78^{* *}$ & $0.75^{* *}$ & $0.83 * *$ & $0.70 * *$ & $0.80 * *$ & $0.85^{* *}$ & 0.71 ** & $0.90 * *$ & $0.83 * *$ & $0.80 * *$ & 0.80 ** \\
\hline
\end{tabular}




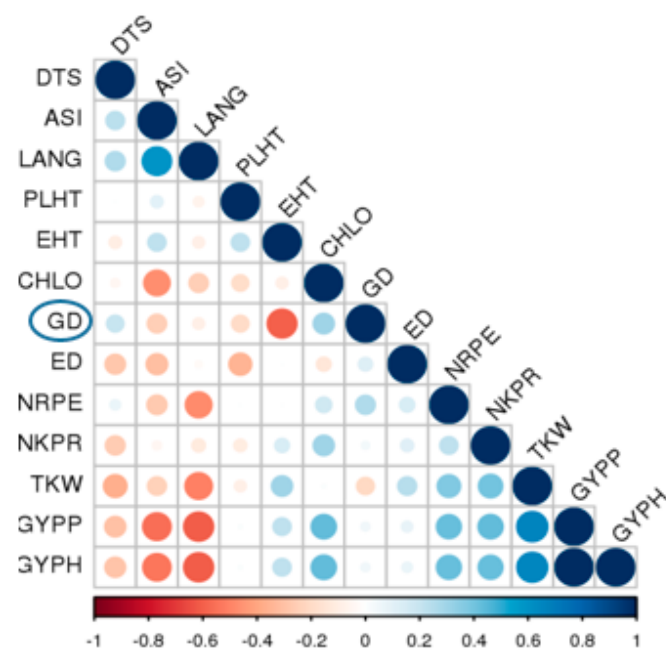

(A)

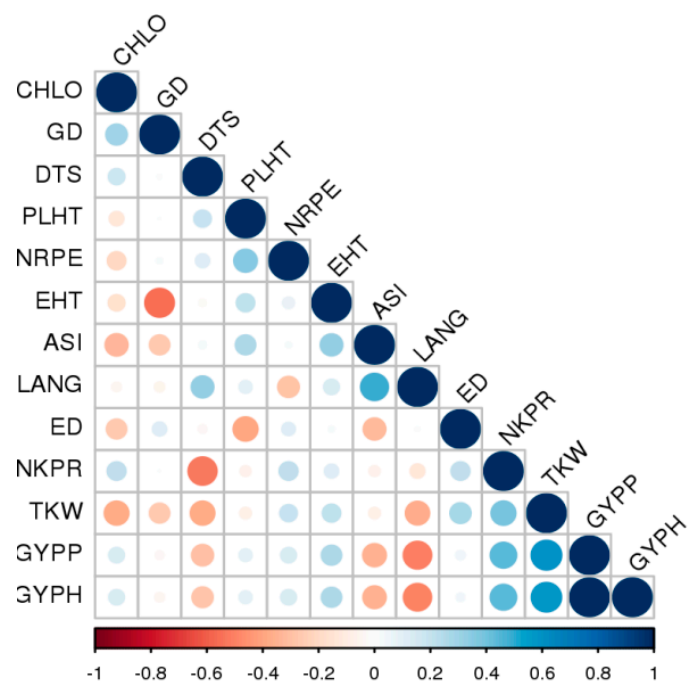

(B)

Figure 4. Corrplot depicting correlation coefficient of genetic distance based on molecular data with $\mathrm{F}_{1}$ hybrid performance (A) and SCA (B) for all studied traits. GD: genetic distance, DTS: days to $50 \%$ silking, ASI: anthesis-silking interval, PLHT: plant height, EHT: ear height, LANG: leaf angle, CHLC: chlorophyll content, ED: ear diameter, NRPE: number of rows per ear, NKPR: number of kernels per row, TKW: thousand kernel weight, GYPP: grain yield per plant and GYPH: grain yield per hectare.

\section{Discussion}

\subsection{Analysis of Variance and Hybrid Performance}

The significant mean squares of L, D and $\mathrm{H}$ observed for all the studied characteristics (Table 2), indicate that the tested locations and densities were dissimilar and there were adequate genetic differences among the hybrids for effective selection of all the studied traits. Significant differences among maize hybrids under different plant densities were also reported [10,35-37]. The presence of significant mean squares for $\mathrm{H} \times \mathrm{D}$ interaction, indicated inconsistent performance of the hybrids across plant densities. In that context, the ranks of maize hybrids differed from one density to another for all measured traits. Therefore, selection of hybrids under various plant densities may be a promising strategy to improve the adaptation of maize hybrids to higher plant density. These results are consistent with the findings of other studies $[12,13,36,38]$.

The significant GCA and SCA effects imply that both additive and non-additive gene effects are involved in governing all traits. The inheritance of a specific trait could be identified based on the ratio of GCA/SCA variances. In the present study, the GCA/SCA ratio was greater than unity for all evaluated characteristics, except NKPR, which indicated the preponderance of additive gene effects in controlling the inheritance of all measured traits, except NKPR which was mainly controlled by non-additive gene action. Therefore, selection breeding methods can be effective for improvement of these traits. This finding is in agreement with that of Mason and Zuber [25] and Al-Naggar et al. [7], who reported that additive genetic effects were important in the inheritance of grain yield and other agronomic traits under different plant densities. However, this result is in contrast to the findings of other studies [36,39], who reported that non-additive gene effects were found to be more important in controlling grain yield inheritance under varying plant densities.

The significant GCA $\times$ L and GCA $\times$ D interactions mean squares for most traits in the present study indicate that the GCA effects of the inbred lines varied significantly under different environments. This result is in agreement with the findings of several authors $[17,26,40,41]$. Likewise, the significant $\mathrm{SCA} \times \mathrm{L}$ and SCA $\times \mathrm{D}$ interactions observed for most traits implied that the performance of the hybrids was not consistent under varying research environments. This suggests the need for extensive 
evaluation of the hybrids in multiple environments in order to identify high yielding and most stable hybrids tolerant to high plant densities [39].

The highest GYPP of all evaluated hybrids in this study was observed under low density (D1), where competition between plants is minimum [12]. As planting density increases, resources to each plant (water, nutrients and light interception) decrease, increasing plant-plant competition and in turn reducing the assimilated supply to developing cobs and, consequently, resulting in a reduction in grain yield per plant [42-44]. The observed reduction in GYPP due to elevating plant density from D1 to D2 and D3 in this study could be a result of the reduction in all yield attributesED, NRPE, NKPR and TKW. These results are consistent with Tang et al. [45], who stated that increasing plant density in maize leads to a reduction in ear diameter, grains per ear, thousand kernels weight and finally single plant yield. Hashemi et al. [46] also demonstrated that grain yield per plant and all yield components linearly decreased with increasing plant density. Moreover, increasing plant density also reduced LANG and CHLC. The decrease in the leaf angle and chlorophyll content in response to high plant density has also been reported previously in maize [13,47,48].

On the other hand, high plant density (D3) caused significant increases in DTS, ASI, PLHT, EHT and GYPH compared with the low density (D1). Delayed silking and increased ASI period, as symptoms of intense interplant competition for growth resources, can be associated with significant yield reductions [15,49]. Increasing plant density initiated greater stress during pollination that can lead to increasing kernel abortions and decreasing grain fill $[8,11]$. These two traits (early DTS and short ASI) could be effective indicators for selecting high density tolerance hybrids [50]. The increased values of PLHT and EHT might be related to the stress imposed on maize plants due to competition for light resulting from elevated plant density which potentially increase stem elongation [51,52]. The increase in GYPH with increasing plant density is largely attributed to the higher number of plants per unit area. This suggested that the increase in GYPH due to increased plant density may offset the reduction in GYPP due to competition between plants. These results are in accordance with the results reported in other studies $[10,12,53,54]$.

The two hybrids $\mathrm{L}_{2} \times \mathrm{L}_{5}$ and $\mathrm{L}_{2} \times \mathrm{L}_{8}$ had the highest GYPP and GYPH under three plant densities, and could be considered the most highly responsive and tolerant to high plant density. Interestingly, the hybrid $\mathrm{L}_{2} \times \mathrm{L}_{8}$ significantly outyielded the check hybrid SC128 under all densities; moreover, it had outstanding features, such as short ASI, short plant and ear position, erect leaf under high plant density. Therefore, this hybrid should be tested extensively in multilocation trials and promoted for adoption to high plant density tolerance. Similar to our results, Al-Naggar et al. [12] reported that the selection of hybrids with high grain yield, better plant and ear heights, short ASI, and erect leaf under high plant density stress is important for the development of tolerant hybrids to high plant densities.

\subsection{GCA and SCA Estimates}

Combining ability analysis helps in the identification of parents with good GCA effects and hybrids with good SCA effects [23]. Selection of parents giving good-performing hybrids is one of the challenges facing breeders. Parents with desirable GCA effect for the target traits can be used to accumulate favorable alleles by recombination and selection [55]. In the current study, high GCA values for the evaluated traits were scattered among the eight inbred lines and changed across plant densities, demonstrating the effects of plant densities on GCA values. Moreover, none of the inbred lines exhibited significant GCA effects for all the measured traits under any of the testing densities. Similar results were reported by other researchers [56,57]. The significant and negative GCA effects were displayed by the inbreds $\mathrm{L}_{1}$ and $\mathrm{L}_{3}$ for DTS and $\mathrm{L}_{1}, \mathrm{~L}_{2}$ and $\mathrm{L}_{5}$ for ASI across the three plant densities, indicating that, these inbreds could be good combiners and possessed favorable alleles towards earliness. Likewise, inbred lines $\mathrm{L}_{5}$ and $\mathrm{L}_{8}$ were the best general combiners for reduced plant and ear heights which are important for lodging tolerance especially under high plant density. The inbred line $\mathrm{L}_{2}$ had the highest positive GCA values for CHLC, NRPE, NKPR and TKW suggesting that this line could be good combiner for improving these traits. Moreover, the best general combiners for 
GYPP and GYPH were $\mathrm{L}_{1}, \mathrm{~L}_{2}$, and $\mathrm{L}_{5}$ under the three plant densities and $\mathrm{L}_{8}$ under D3. These inbreds could transfer desirable alleles for improved grain yield to their progenies to develop hybrids tolerant to high plant density. The superiority of these inbreds in GCA effects for grain yield was associated with their superiority in GCA effects for some other traits. Interestingly, the inbred line $\mathrm{L}_{1}$, which had desirable GCA effects for GYPP and GYPH, was also found to be good a general combiner for earliness, short ASI, short PLHT, reduced LANG and increased TKW. Previous findings proved that positive GCA effects for grain yield and negative GCA effects for DTS, PLHT, and LANG traits are a good indicator of high plant density tolerance [13]. Thus, the inbred line $\mathrm{L}_{1}$ has potential to be used to improve maize grain yield under high plant density.

Estimates of SCA effects provide important information about the non-additive gene effects (dominance and epistatic interaction), which can also be related to hybrid vigor, assisting in the selection of the best hybrid combinations [58]. The highly positive and significant SCA effects for grain yield and its components indicated that the produced hybrids were good specific combiners for developing high-yielding hybrids [1]. In the present study, the most promising specific combiners for grain yield (GYPP or GYPH) and some of its components were $\mathrm{L}_{1} \times \mathrm{L}_{3}, \mathrm{~L}_{1} \times \mathrm{L}_{6}, \mathrm{~L}_{2} \times \mathrm{L}_{5}, \mathrm{~L}_{2} \times \mathrm{L}_{8}$, $\mathrm{L}_{4} \times \mathrm{L}_{5}$ and $\mathrm{L}_{7} \times \mathrm{L}_{8}$ under the three plant densities. These hybrids involved at least one high GCA parent, which could be exploited by conventional breeding procedures. This finding is in line with the result reported in other studies [56,59]. In their studies, high SCA was observed in cross combinations involving one line with high GCA and another with low GCA effects.

Two hybrids, $\mathrm{L}_{2} \times \mathrm{L}_{5}$ and $\mathrm{L}_{2} \times \mathrm{L}_{8}$, had desirable significant positive SCA coupled with high mean grain yield under the three plant densities, revealing good correspondence between mean grain yield and SCA effects [1]. Regardless of their significant SCA effects, three crosses $\mathrm{L}_{3} \times \mathrm{L}_{4}, \mathrm{~L}_{3} \times \mathrm{L}_{6}$ and $\mathrm{L}_{6}$ $\times \mathrm{L}_{7}$, constituted from parents with low $\times$ low GCA effects for GYPP and GYPH were not favorable due to insufficient additive variance. This indicates that both GCA and SCA should be taken into consideration in the selection of elite parents for the development of heterotic hybrids [18]. It is notable that none of the hybrids exhibited significant SCA effects for all the traits. However, the hybrids $\mathrm{L}_{2}$ $\times \mathrm{L}_{5}, \mathrm{~L}_{2} \times \mathrm{L}_{8}$ and $\mathrm{L}_{4} \times \mathrm{L}_{5}$ were found to be good specific combiners for more than one trait, such as ASI, LANG, TKW, GYPP and GYPH. Accordingly, these hybrids would be useful to increase maize grain yield under high plant density for their complementary characteristics, including, short ASI, erect leaf and high grain yield under high plant density. In concordance with the findings reported here, desirable significant SCA under high plant density for ASI, LANG and grain yield has previously been reported by Al-Naggar et al. [13].

\subsection{SSR Polymorphisms, Genetic Distance (GD) and Cluster Analysis}

The mean number of alleles (2.7) per locus obtained in this study was close to the values reported by other researchers $[26,27,34]$, who detected averages of $2.9,2.57$ and 3.0 alleles per locus, respectively. However, it was lower than the 6.21 alleles/locus reported by Oppong et al. [60] or the 5.7 alleles/locus found by Oyekunle et al. [61] in maize inbred lines using SSR markers. The differences in the means of alleles among different studies could be attributed to the differences in sample size, repeat length and number of the SSR markers involved in the studies [27]. The lower values observed in this study could arise from the small number of lines used for genotyping.

The PIC demonstrates the informativeness of the SSR loci and their potential to detect differences among the inbred lines based on their genetic relationships [62]. Informative markers can be categorized as highly informative (PIC $>0.5)$, reasonably informative $(0.5<$ PIC $<0.25)$ and slightly informative (PIC < 0.25), as reported by Botstein et al. [63]. Accordingly, four markersumc1014, phi112, phi015 and umc1033 with high PIC values, and hence high discriminatory power, were identified. The average gene diversity (0.50) detected among the tested inbred lines in this study indicated high levels of polymorphisms within the inbred lines. This result is in close agreement with the findings reported in other studies [30,64]. The frequency of the most common (major) alleles had an average of 0.59 , suggesting that $59.0 \%$ of the studied inbreds shared a common major allele at any of the tested loci. 
Assessing the genetic diversity is essential for enhancing the yield and conservation strategies of main crops [65-70], such as maize that has high an economic importance [71]. The average genetic diversity existing among all the inbred lines was relatively high (0.61). This indicated that there was considerable genetic diversity among the inbreds based on the microsatellite markers analysis [72]. The largest GD in this study was between the Egyptian (local) and CIMMYT (exotic) inbred lines. The relatively large genetic distance between local and exotic lines, suggesting the opportunity to use these lines for the development of high-yielding and stress-tolerant hybrids. Indeed, the two high-yielding hybrids $\left(\mathrm{L}_{2} \times \mathrm{L}_{5}\right.$ and $\left.\mathrm{L}_{2} \times \mathrm{L}_{8}\right)$ under the three plant densities consisted of local $\times$ exotic line combinations. This indicates that novel and complementary alleles existing in the germplasm from the two countries can be exploited for superior maize hybrid development and population improvement [73]. Moreover, it implies the potential benefits of exchanging germplasm between breeding programs for the development of high yielding and density tolerant hybrids.

The dendrogram constructed using the UPGMA clustering grouped the inbred lines into two main clusters, which generally agreed with their origin. One cluster was composed of CIMMYT inbred lines, while the other consisted of local inbreds. This result is consistent with the findings of Mageto et al. [17], who reported that clustering based on GD grouped maize inbred lines according to their origin. Similarly, [34,64] revealed the effectiveness of SSR markers for classifying maize inbreds according to their origin in their studies.

\subsection{Association between Genetic Distance, F1 Hybrid Performance and SCA}

Our results showed that GD of the parental inbreds was not significantly correlated with the mean of $F_{1}$ hybrids for any of the evaluated traits across the tested environments. This implied that the SSR-based GD could not be used to predict the performance of $F_{1}$ hybrids in this study. This result is consistent with those reported by $[26,33,34,40]$. Bernardo [74] attributed this poor correlation to the lack of linkage between genes controlling the trait and markers used to estimate GD, inadequate genome coverage and different levels of dominance among hybrids. Contrary to the current finding, a significant correlation was reported between molecular GD and $F_{1}$ hybrid performance [32,75]. There was no significant correlation between GD and SCA for all the traits, suggesting that SSR-based GD might not be effective in predicting SCA effects in the studied materials. Similarly, non-significant association between genetic distances and SCA was reported by [40,76]. However, Betran et al. [75] reported a significant correlation between GD and SCA for maize grain yield. Furthermore, our results showed that SCA effects were significantly correlated with $\mathrm{F}_{1}$ hybrid performance for all the traits. This indicated that SCA could be used to predict the performance of $\mathrm{F}_{1}$ hybrids. This result is in agreement with the findings of $[17,26]$.

\section{Conclusions}

This study revealed a considerable variability among $\mathrm{F}_{1}$ hybrids for all traits under different plant densities. Additive and non-additive gene effects are involved in the genetic control of all traits, with a predominance of the additive gene action for most traits. Selection of potential hybrids for density tolerance breeding programs should be based on both GCA and SCA effects. The inbred lines $\mathrm{L}_{1}$ and $\mathrm{L}_{3}$ were identified as excellent combiners for earliness, $\mathrm{L}_{5}$ and $\mathrm{L}_{8}$ for reduced plant and ear heights and $\mathrm{L}_{1}, \mathrm{~L}_{2}$, and $\mathrm{L}_{5}$ for increased grain yield under varying plant densities. The best hybrids $\mathrm{L}_{2} \times \mathrm{L}_{5}$ and $\mathrm{L}_{2}$ $\times \mathrm{L}_{8}$ for grain yield and other multiple traits were identified for further evaluation. The estimated GD based on SSR markers in this study could not be used to predict the hybrids performance and SCA effects. Nevertheless, SCA could be used to predict the hybrids performance across all plant densities. Although SSR determined that GD was not useful in predicting hybrid performance and SCA effects, it was effective in classifying the inbred lines according to their origin, signifying the efficiency of SSR marker for diversity and clustering analyses. The findings of the present study might have important implications for breeding programs designed to improve density tolerance in maize. 


\section{Materials and Methods}

\subsection{Plant Materials}

Eight white maize (Zea mays L.) inbred lines showing clear differences in grain yield and other agronomic characteristics were chosen as parents in this study. Four inbreds $\left(\mathrm{L}_{1}, \mathrm{~L}_{2} \mathrm{~L}_{3}\right.$ and $\left.\mathrm{L}_{4}\right)$ were obtained from Maize Research Department, Agricultural Research Center (ARC) in Egypt and the other four $\left(\mathrm{L}_{5}, \mathrm{~L}_{6}, \mathrm{~L}_{7}\right.$ and $\left.\mathrm{L}_{8}\right)$ were introduced from the International Maize and Wheat Improvement Center (CIMMYT). The parental codes, names and sources of these inbred lines are listed in Table 8.

Table 8. Code, name and source of the parental maize inbred lines.

\begin{tabular}{ccc}
\hline Parent Code & Name & Source \\
\hline L1 & IL36 & ARC-Egypt \\
L2 & IL94 & ARC-Egypt \\
L3 & IL53 & ARC-Egypt \\
L4 & IL38 & ARC-Egypt \\
L5 & CML538 & CIMMYT-Mexico \\
L6 & CML203 & CIMMYT-Mexico \\
L7 & CML206 & CIMMYT-Mexico \\
L8 & CML441 & CIMMYT-Mexico \\
\hline
\end{tabular}

\subsection{Production and Evaluation of $F_{1}$ Hybrids}

In the 2017 season, all possible diallel crosses (excluding reciprocals) were made among the eight inbred lines to obtain seeds of $28 \mathrm{~F}_{1}$ hybrids. In the 2018 season, the resulting $28 \mathrm{~F}_{1}$ white hybrids plus the commercial check hybrid SC128 were evaluated under three plant densities, i.e., 59,500 (D1), 71,400 (D2) and 83,300 (D3) plants ha ${ }^{-1}$ at two locations. The two locations were El-Mahmoudia, El-Behira, Egypt $\left(\left(31^{\circ} 3^{\prime} \mathrm{N}, 30^{\circ} 48^{\prime} \mathrm{E}\right)\right)$ in a private farm, and the Experimental Farm, Faculty of Agriculture, Kafrelsheikh University, Egypt ( $\left.\left(31^{\circ} 6^{\prime} \mathrm{N}, 30^{\circ} 56^{\prime} \mathrm{E}\right)\right)$. A split-plot design in randomized complete blocks (RCB) arrangement with three replications was used in each location. The three plant densities were located at the main plots, while the hybrids were located at the sub plots. Each subplot consisted of one ridge of $6 \mathrm{~m}$ long and $0.7 \mathrm{~m}$ width. Two seeds were sown in hills at 24, 20 and $17 \mathrm{~cm}$ apart, and thereafter (before the 1st irrigation) were thinned to one plant/hill to achieve the three plant densities, i.e., D1, D2 and D3, respectively. Phosphorus at the rate of $476 \mathrm{~kg} \mathrm{ha}^{-1}$ in the form of calcium super phosphate $\left(15.5 \% \mathrm{P}_{2} \mathrm{O}_{5}\right)$ was added to the soil during seedbed preparation, and potassium sulphate $\left(48 \% \mathrm{~K}_{2} \mathrm{O}\right)$ at a level of $120 \mathrm{~kg} \mathrm{ha}^{-1}$ was applied after thinning. Moreover, nitrogen at the rate of $286 \mathrm{~kg}$ $\mathrm{ha}^{-1}$ was added in two equal doses before the first and second irrigation. All other standard agronomic practices including weed control were followed in each location. Soil analysis was conducted on soil samples collected from $30 \mathrm{~cm}$ depth from each location according to Association of Officinal Analytical Chemists (A.O.A.C 2005) [77] (Supplementary Materials, Table S2). Additionally, the meteorological data are presented in the Supplementary Materials, Figure S1.

\subsection{Data Collection}

Data were collected on days to 50\% silking (DTS, days from the planting to 50\% extrusion of silks from the plants), anthesis-silking interval (ASI, calculated as the difference between days to $50 \%$ silking and days to 50\% anthesis), plant height (PLHT, measured in $\mathrm{cm}$ as the distance from the soil surface to the top of the first tassel branch) and ear height (EHT, measured in $\mathrm{cm}$ as the distance from the soil surface to the base of the topmost ear). Leaf angle (LANG) $\left(^{\circ}\right)$ was measured as the angle between stem and blade of the leaf just above ear leaf. Chlorophyll content (CHLC, SPAD units) was measured by hand-held chlorophyll meter (SPAD-502; Minolta Sensing Co., Ltd., Hangzhou, Japan) from the leaf of the top-most ear. The LANG and CHLC values were recorded on ten guarded plants within each plot, and then the values were averaged per plot. At harvest, ear diameter (ED), number of 
rows per ear (NKPR), number of kernels per row (NKPR), thousand kernel weight (TKW), grain yield per plant (GYPP, in g plant ${ }^{-1}$ ) and grain yield per hectare (GYPH, in ton ha ${ }^{-1}$ ) were estimated. Plots were hand-harvested, and the weight of the shelled grain (adjusted to 15.5\% grain moisture content) was used to calculate GYPP and GYPH. Grain moisture at harvest was measured using a hand-held moisture meter.

\subsection{Molecular Analysis}

\subsubsection{DNA Isolation}

Leaves were sampled from 10 to 15 seedlings of each inbred line after twenty days from planting. Genomic DNA was isolated using CTAB method [78]. DNA quantity as well as quality was assessed using NanoDrop spectrophotometer (ND-1000, USA).

\subsubsection{SSR Primers and PCR Amplification}

Twenty-two SSR markers were randomly selected from the MaizeGDB database (www.maizegdb. org). The 22 primer pairs were tested to identify the polymorphic ones. Only ten markers were found to be polymorphic and they used for the SSR analysis (Supplementary Materials, Table S3). PCR was performed in a volume of $10 \mu \mathrm{L}$ reaction mixture containing $1 \mu \mathrm{L}$ of $20 \mathrm{ng} / \mu \mathrm{L}$ genomic DNA template, 1 unit Taq DNA polymerase (Promega, Madison, WI, USA), $2 \mathrm{mM} \mathrm{MgCl2,} 0.2 \mathrm{mM}$ dNTPs and 0.5 $\mu \mathrm{M}$ of reverse and forward primer. The PCR reaction was initially started by denaturation at $94{ }^{\circ} \mathrm{C}$ for $2 \mathrm{~min}$, followed by 35 cycles consisting of denaturation at $94^{\circ} \mathrm{C}$ for $30 \mathrm{sec}, 30 \mathrm{sec}$ of annealing at $55{ }^{\circ} \mathrm{C}, 30 \mathrm{sec}$ of extension at $72{ }^{\circ} \mathrm{C}$ and a final extension of $3 \mathrm{~min}$ at $72{ }^{\circ} \mathrm{C}$. Amplified products were electrophoresed on 1.5\% agarose gel. The gels were stained with ethidium bromide and then distained with tap water and photographed using gel documentation system (UVITEC, Cambridge, UK).

\subsection{Statistical Analysis}

Analysis of variance (ANOVA) was computed for all data using SAS software (SAS Institute Inc, 2008). Combined analysis of variance of the split-plot design across the two locations was performed if the homogeneity test was non-significant. Least significant difference (LSD) values were calculated to test the significance of differences between means according to Steel et al. [79]. General combining ability (GCA) effects of the parents and specific combining ability (SCA) effects of the hybrids as well as their mean squares were computed according to Griffing's method 4 model I [80], using the DIALLEL-SAS program [81]. The testing of significance of GCA and SCA effects was done at $5 \%$ and $1 \%$ probability. Pearson's coefficients of correlation $(\mathrm{r})$ were calculated and plotted using the package corrplot [82]. Based on the mean of each trait the reduction or increase due to increased plant density was calculated as follow:

$$
\text { Change } \%=100(\mathrm{D} 2 \text { or D3 }-\mathrm{D} 1) / \mathrm{D} 1
$$

\subsection{SSR Data Analysis}

The amplified bands were scored for each SSR marker based on the presence or absence of bands, generating a binary data matrix of (1) and (0) for each marker. The number of alleles per locus, major allele frequency, gene diversity and polymorphic information content (PIC) were calculated to assess allele diversity of each marker. The value of polymorphic information content (PIC) of each SSR marker was determined as described by Botstein et al. [63] as follows:

$$
1-\sum_{i=1}^{n} \mathrm{P}_{j}^{2}-\sum_{i=1}^{n-1} \sum_{j=i+1}^{n} 2 \mathrm{P}_{i}^{2} \mathrm{P}_{j}^{2}
$$

where $\mathrm{P}_{i}$ and $\mathrm{P}_{j}$ are the frequencies of the $i$ th and $j$ th allele of a given marker, respectively. 
Genetic distances between pairs of inbred lines were calculated according to [83], using the PAST program. The dendrogram tree was generated with the unweighted pair group method using arithmetic averages (UPGMA) by the computational package MVSP version 3.1.

Supplementary Materials: The following are available online at http://www.mdpi.com/2223-7747/9/9/1140/s1. Table S1: Mean performance of the $28 \mathrm{~F}_{1}$ crosses and the check hybrid SC128 for all the studied traits under the three plant densities across the two locations. Table S2: Physical and chemical soil properties for the two locations during 2018 season. Table S3: List of SSR primers and their sequences used in the present study. Figure S1: Daily maximum temperature ( $\mathrm{T}$ max), minimum temperature ( $\mathrm{T} \mathrm{min}$ ) and solar radiation (SRAD) for the two locations during 2018 season.

Author Contributions: M.M.K., M.R., K.M.I., A.S.A., M.M.E., A.M.S.K., M.A.E.-E., and E.M.H. designed the study, performed the experiments, analyzed the data and wrote the manuscript. All authors have read and agreed to the published version of the manuscript.

Funding: This research received no external funding.

Acknowledgments: Faculty of agriculture, Kafrelsheikh University, Egypt, is thankfully acknowledged for carrying out this work. Tanta University in Egypt is also thankfully acknowledged for the support provided for conducting this work. The Agricultural Research Center (ARC) in Egypt and the International Maize and Wheat Improvement Center (CIMMYT), are thankfully acknowledged for providing us the seeds of the inbred lines used in this study.

Conflicts of Interest: The authors declare no conflict of interest.

\section{References}

1. Elmyhun, M.; Liyew, C.; Shita, A.; Andualem, M. Combining ability performance and heterotic grouping of maize (Zea mays) inbred lines in testcross formation in Western Amhara, North West Ethiopia. Cogent Food Agric. 2020, 6, 1727625. [CrossRef]

2. FAOSTAT. Food and Agriculture Organization of the United Nations Statistics Division. Available online: http://faostat.fao.org/site/567/DesktopDefault.aspx (accessed on 23 February 2020).

3. Zohry, A.; Ouda, S.; Noreldin, T. Solutions for maize production consumption gap in Egypt. In Proceedings of the 4th African Regional ICID Conference, Aswan, Egypt, 26-28 April 2016; pp. 24-28.

4. Abdelaal, H.S.A.; McFadden, D.T. Grains production prospects and long run food security in Egypt. Sustainability 2019, 11, 4457. [CrossRef]

5. Shao, H.; Shi, D.; Shi, W.; Ban, X.; Chen, Y.; Ren, W.; Chen, F.; Mi, G. Nutrient accumulation and remobilization in relation to yield formation at high planting density in maize hybrids with different senescent characters. Arch. Agron. Soil Sci. 2020, 1-17. [CrossRef]

6. Zhao, J.R.; Wang, R.H. Factors promoting the steady increase of American maize production and their enlightenments for China. J. Maize Sci. 2009, 17, 156-159.

7. Al-Naggar, A.; Atta, M. Elevated plant density effects on performance and genetic parameters controlling maize (Zea mays L.) agronomic traits. J. Adv. Biol. Biotechnol. 2017, 12, 1-20. [CrossRef]

8. Andrade, F.H.; Vega, C.R.; Uhart, S.; Cirilo, A.; Cantarero, M.; Valentinuz, O. Kernel number determination in maize. Crop. Sci. 1999, 39, 453-459. [CrossRef]

9. Edmeades, G.O.; Bolaños, J.; Elings, A.; Ribaut, J.M.; Banziger, M.; Westgate, M.E.; Westgate, M.; Boote, K. The role and regulation of the anthesis-silking interval in maize. Physiol. Model. Kernel Set Maize 2015, 29, 43-73. [CrossRef]

10. Sangoi, L.; Gracietti, M.; Rampazzo, C.; Bianchetti, P. Response of Brazilian maize hybrids from different eras to changes in plant density. Field Crop. Res. 2002, 79, 39-51. [CrossRef]

11. Echarte, L.; Luque, S.L.; Andrade, F.H.; Sadras, V.; Cirilo, A.; Otegui, M.E.; Vega, C.R. Response of maize kernel number to plant density in Argentinean hybrids released between 1965 and 1993. Field Crop. Res. 2000, 68, 1-8. [CrossRef]

12. Al-Naggar, A.; Shabana, R.A.; Atta, M.M.; Al-Khalil, T.H.; Al-Naggar, A.M.M. Maize response to elevated plant density combined with lowered N-fertilizer rate is genotype-dependent. Crop. J. 2015, 3, 96-109. [CrossRef]

13. Al-Naggar, A.M.M.; Atta, M.M.; Ahmed, M.; Younis, A. Mean performance, heterobeltiosis and combining ability of corn (Zea mays L.) agronomic and yield traits under elevated plant density. J. Appl. Life Sci. Int. 2016, 7, 1-20. [CrossRef] [PubMed] 
14. Hammer, G.L.; Dong, Z.; McLean, G.; Doherty, A.; Messina, C.; Schussler, J.; Zinselmeier, C.; Paszkiewicz, S.; Cooper, M. Can changes in canopy and/or root system architecture explain historical maize yield trends in the U.S. corn belt? Crop. Sci. 2009, 49, 299-312. [CrossRef]

15. Tokatlidis, I.; Koutroubas, S. A review of maize hybrids' dependence on high plant populations and its implications for crop yield stability. Field Crop. Res. 2004, 88, 103-114. [CrossRef]

16. Ruffo, M.L.; Gentry, L.; Henninger, A.S.; Seebauer, J.R.; Below, F.E. Evaluating management factor contributions to reduce corn yield gaps. Agron. J. 2015, 107, 495-505. [CrossRef]

17. Mageto, E.K.; Makumbi, D.; Njoroge, K.; Nyankanga, R. Genetic analysis of early-maturing maize (Zea mays L.) inbred lines under stress and nonstress conditions. J. Crop. Improv. 2017, 31, 560-588. [CrossRef]

18. Kamara, M.M.; El-Degwy, I.S.; Koyama, H. Estimation combining ability of some maize inbred lines using line $\mathrm{x}$ tester mating design under two nitrogen levels. Aust. J. Crop Sci. 2014, 8, 1336.

19. Carena, M.J.; Hallauer, A.R.; Filho, J.M. Quantitative Genetics in Maize Breeding; Springer: New York, NY, USA, 2010.

20. Henry, W.B.; Blanco, M.H.; Rowe, D.E.; Windham, G.L.; Murray, S.C.; Williams, W.P. Diallel analysis of diverse maize germplasm lines for agronomic characteristics. Crop. Sci. 2014, 54, 2547-2556. [CrossRef]

21. Sughroue, J.R.; Hallauer, A.R. Analysis of the diallel mating design for maize inbred lines. Crop. Sci. 1997, 37, 400-405. [CrossRef]

22. Zhang, Y.; Fan, X.; Yao, W.; Piepho, H.P.; Kang, M.S. Diallel analysis of four maize traits and a modified heterosis hypothesis. Crop. Sci. 2016, 56, 1115-1126. [CrossRef]

23. Sprague, G.F.; Tatum, L.A. General vs. specific combining ability in single crosses of corn1. Agron. J. 1942, 34, 923-932. [CrossRef]

24. Al-Naggar, A.; Atta, M.; Shabana, R.; Al-Khalil, T. Heterosis and type of gene action for some adaptive traits to high plant density in maize. Egypt. J. Plant. Breed. 2014, 18, 189-209. [CrossRef]

25. Mason, L.; Zuber, M.S. Diallel analysis of maize for leaf angle, leaf area, yield, and yield components 1. Crop. Sci. 1976, 16, 693-696. [CrossRef]

26. Badu-Apraku, B.; Oyekunle, M.; Fakorede, M.A.B.; Vroh, I.; Akinwale, R.O.; Aderounmu, M. Combining ability, heterotic patterns and genetic diversity of extra-early yellow inbreds under contrasting environments. Euphytica 2013, 192, 413-433. [CrossRef]

27. Akinwale, R.; Badu-Apraku, B.; Fakorede, M.; Vroh-Bi, I. Heterotic grouping of tropical early-maturing maize inbred lines based on combining ability in Striga-infested and Striga-free environments and the use of SSR markers for genotyping. Field Crop. Res. 2014, 156, 48-62. [CrossRef]

28. Akaogu, I.C.; Badu-Apraku, B.; Adetimirin, V.O.; Vroh-Bi, I.; Oyekunle, M.; Akinwale, R.O. Genetic diversity assessment of extra-early maturing yellow maize inbreds and hybrid performance in Striga-infested and Striga-free environments. J. Agric. Sci. 2013, 151, 519-537. [CrossRef]

29. Nyaligwa, L.; Hussein, S.; Amelework, B.; Ghebrehiwot, H. Genetic diversity analysis of elite maize inbred lines of diverse sources using SSR markers. Maydica 2015, 60, M29.

30. Sserumaga, J.P.; Ji, H.; Njoroge, K.; Muthomi, J.; Chemining'wa, G.; Si-myung, L.; Kim, H.; Asea, G.; Makumbi, D. Molecular characterization of tropical maize inbred lines using microsatellite DNA markers. Maydica 2014, 59, 267-274.

31. Phumichai, C.; Doungchan, W.; Puddhanon, P.; Jampatong, S.; Grudloyma, P.; Kirdsri, C.; Chunwongse, J.; Pulam, T. SSR-based and grain yield-based diversity of hybrid maize in Thailand. Field Crop. Res. 2008, 108, 157-162. [CrossRef]

32. Singh, P. Genetic distance, heterosis and combing ability studies in maize for predicting $\mathrm{F}_{1}$ hybrid performance. Sabrao J. Breed. Genet. 2015, 47, 21-28.

33. Dhliwayo, T.; Pixley, K.; Menkir, A.; Warburton, M. Combining ability, genetic distances, and heterosis among elite CIMMYT and IITA tropical maize inbred lines. Crop. Sci. 2009, 49, 1201-1210. [CrossRef]

34. Menkir, A.; Melake-Berhan, A.; The, C.; Ingelbrecht, I.; Adepoju, A. Grouping of tropical mid-altitude maize inbred lines on the basis of yield data and molecular markers. Theor. Appl. Genet. 2004, 108, 1582-1590. [CrossRef] [PubMed]

35. Lashkari, M.; Madani, H.; Ardakani, M.R.; Golzardi, F.; Zargari, K. Effect of plant density on yield and yield components of different corn (Zea mays L.) hybrids. Am-Euras J. Agric. Environ. Sci. 2011, 10, 450-457.

36. Mansfield, B.D.; Mumm, R.H. Survey of plant density tolerance in U.S. maize germplasm. Crop. Sci. 2014, 54, 157-173. [CrossRef] 
37. Trachsel, S.; Vicente, F.M.S.; Suarez, E.A.; Rodriguez, C.S.; Atlin, G.N. Effects of planting density and nitrogen fertilization level on grain yield and harvest index in seven modern tropical maize hybrids (Zea mays L.). J. Agric. Sci. 2015, 154, 689-704. [CrossRef]

38. Kamara, A.Y.; Menkir, A.; Abubakar, A.W.; Tofa, A.I.; Ademulegun, T.D.; Omoigui, L.O.; Kamai, N. Maize hybrids response to high plant density in the Guinea savannah of Nigeria. J. Crop. Improv. 2020, 1-20. [CrossRef]

39. Abd El-Aty, M.; El-Hity, M.; Amer, E.; El-Mouslhy, T. Selection of maize (Zea mays) hybrids for plant density tolerance. Indian J. Agric. Sci. 2019, 89, 951-957.

40. Makumbi, D.; Betrán, J.F.; Bänziger, M.; Ribaut, J.M. Combining ability, heterosis and genetic diversity in tropical maize (Zea mays L.) under stress and non-stress conditions. Euphytica 2011, 180, 143-162. [CrossRef]

41. Badu-Apraku, B.; Oyekunle, M. Genetic analysis of grain yield and other traits of extra-early yellow maize inbreds and hybrid performance under contrasting environments. Field Crop. Res. 2012, 129, 99-110. [CrossRef]

42. Liu, W.; Tollenaar, M. Response of yield heterosis to increasing plant density in maize. Crop. Sci. 2009, 49, 1807-1816. [CrossRef]

43. Tollenaar, M.; Wu, J. Yield improvement in temperate maize is attributable to greater stress tolerance. Crop. Sci. 1999, 39, 1597-1604. [CrossRef]

44. Van Averbeke, W.; Marais, J.N. Maize response to plant population and soil water supply: I. Yield of grain and total above-ground biomass. S. Afr. J. Plant. Soil 1992, 9, 186-192. [CrossRef]

45. Tang, L.; Ma, W.; Noor, M.A.; Li, L.; Hou, H.; Zhang, X.; Zhao, M. Density resistance evaluation of maize varieties through new "Density-Yield Model" and quantification of varietal response to gradual planting density pressure. Sci. Rep. 2018, 8, 1-16. [CrossRef] [PubMed]

46. Hashemi, A.M.; Herbert, S.J.; Putnam, D.H. Yield response of corn to crowding stress. Agron. J. 2005, 97, 839-846. [CrossRef]

47. Ren, B.; Liu, W.; Zhang, J.; Dong, S.; Liu, P.; Zhao, B. Effects of plant density on the photosynthetic and chloroplast characteristics of maize under high-yielding conditions. Sci. Nat. 2017, 104, 12. [CrossRef]

48. Gou, L.; Xue, J.; Qi, B.; Ma, B.; Zhang, W. Morphological variation of maize cultivars in response to elevated plant densities. Agron. J. 2017, 109, 1443-1453. [CrossRef]

49. Sangoi, L. Understanding plant density effects on maize growth and development: An important issue to maximize grain yield. Ciência Rural 2001, 31, 159-168. [CrossRef]

50. Al-Naggar, A.; Shabana, R.; Hassanein, M.S.; Elewa, T.A.; Younis, A.; Metwally, A. Secondary traits and selection environment of plant density tolerance in maize inbreds and testcrosses. J. Adv. Biol. Biotechnol. 2017, 14, 1-13. [CrossRef]

51. Gyenes-Hegyi, Z.; Pók, I.; Kizmus, L.; Al, E. Plant height and height of the main ear in maize (Zea mays L.) at different locations and different plant densities. Acta Agron. Hung. 2002, 50, 75-84. [CrossRef]

52. Carena, M.; Cross, H. Plant density and maize germplasm improvement in the northern Corn Belt. Maydica 2003, 48, 105-111.

53. Tollenaar, M.; Lee, E. Yield potential, yield stability and stress tolerance in maize. Field Crop. Res. 2002, 75, 161-169. [CrossRef]

54. Duvick, D.N. The Contribution of Breeding to Yield Advances in maize (Zea mays L.). Adv. Agron. 2005, 86, 83-145. [CrossRef]

55. Obeng-Bio, E.; Badu-Apraku, B.; Ifie, B.E.; Danquah, A.; Blay, E.T.; Annor, B. Genetic analysis of grain yield and agronomic traits of early provitamin A quality protein maize inbred lines in contrasting environments. J. Agric. Sci. 2019, 157, 413-433. [CrossRef]

56. Chiuta, N.E.; Charles, M.S. Combining ability of quality protein maize inbred lines for yield and morpho-agronomic traits under optimum as well as combined drought and heat-stressed conditions. Agronomy 2020, 10, 184. [CrossRef]

57. Gissa, D.W.; Zelleke, H.; Labuschagne, M.T.; Hussien, T.; Singh, H. Heterosis and combining ability for grain yield and its components in selected maize inbred lines. S. Afr. J. Plant. Soil 2007, 24, 133-137. [CrossRef]

58. Reif, J.C.; Gumpert, F.M.; Fischer, S.; Melchinger, A.E. Impact of interpopulation divergence on additive and dominance variance in hybrid populations. Genetics 2007, 176, 1931-1934. [CrossRef]

59. Ejigu, Y.G.; Tongoona, P.B.; Ifie, B.E. General and specific combining ability studies of selected tropical white maize inbred lines for yield and yield related traits. Int. J. Agric. Res. Innov. Technol. 2017, 7, 381-396. 
60. Oppong, A.; Bedoya, C.A.; Ewool, M.B.; Asante, M.D.; Thomson, R.N.; Adu-Dapaah, H.; Lamptey, J.N.; Ofori, K.; Offei, S.K.; Warburton, M.L. Bulk genetic characterization of Ghanaian maize landraces using microsatellite markers. Maydica 2014, 59, 1-8.

61. Oyekunle, M.; Badu-Apraku, B.; Hearne, S.; Franco, J. Genetic diversity of tropical early-maturing maize inbreds and their performance in hybrid combinations under drought and optimum growing conditions. Field Crop. Res. 2015, 170, 55-65. [CrossRef]

62. Legesse, B.W.; Myburg, A.; Pixley, K.V.; Botha, A. Genetic diversity of African maize inbred lines revealed by SSR markers. Hereditas 2007, 144, 10-17. [CrossRef]

63. Botstein, D.; White, R.L.; Skolnick, M.; Davis, R.W. Construction of a genetic linkage map in man using restriction fragment length polymorphisms. Am. J. Hum. Genet. 1980, 32, 314-331.

64. Adu, G.B.; Awuku, F.; Amegbor, I.; Haruna, A.; Manigben, K.; Aboyadana, P. Genetic characterization and population structure of maize populations using SSR markers. Ann. Agric. Sci. 2019, 64, 47-54. [CrossRef]

65. El-Esawi, M.; Germaine, K.J.; Bourke, P.; Malone, R. AFLP analysis of genetic diversity and phylogenetic relationships of Brassica oleracea in Ireland. Comptes Rendus Biol. 2016, 339, 163-170. [CrossRef] [PubMed]

66. El-Esawi, M.; Alaraidh, I.A.; Alsahli, A.A.; Ali, H.M.; Alayafi, A.A.; Witczak, J.; Ahmad, M. Genetic variation and alleviation of salinity stress in barley (Hordeum vulgare L.). Molecules 2018, 23, 2488. [CrossRef] [PubMed]

67. El-Esawi, M.; Al-Ghamdi, A.A.; Ali, H.M.; Alayafi, A.A.; Witczak, J.; Ahmad, M. Analysis of genetic variation and enhancement of salt tolerance in French pea (Pisum sativum L.). Int. J. Mol. Sci. 2018, 19, 2433. [CrossRef] [PubMed]

68. El-Esawi, M. Genetic diversity and evolution of Brassica genetic resources: From morphology to novel genomic technologies-A review. Plant. Genet. Resour. 2016, 15, 388-399. [CrossRef]

69. El-Esawi, M.; Sammour, R. Karyological and phylogenetic studies in the genus Lactuca L. (Asteraceae). Cytologia 2014, 79, 269-275. [CrossRef]

70. El-Esawi, M.; Al-Ghamdi, A.A.; Ali, H.M.; Ahmad, M. Overexpression of AtWRKY30 transcription factor enhances heat and drought stress tolerance in wheat (Triticum aestivum L.). Genes 2019, 10, 163. [CrossRef]

71. Vwioko, E.; Adinkwu, O.; El-Esawi, M.A. Comparative physiological, biochemical, and genetic responses to prolonged waterlogging stress in okra and maize given exogenous ethylene priming. Front. Physiol. 2017, 8, 632. [CrossRef]

72. Xia, X.; Reif, J.; Hoisington, D.; Melchinger, A.; Frisch, M.; Warburton, M. Genetic diversity among CIMMYT maize inbred lines investigated with SSR markers. Crop. Sci. 2004, 44, 2230-2237. [CrossRef]

73. Adebayo, M.A.; Menkir, A.; Gedil, M.; Blay, E.; Gracen, V.; Danquah, E.; Funmilayo, L. Diversity assessment of drought tolerant exotic and adapted maize (Zea mays L.) inbred lines with microsatellite markers. J. Crop. Sci. Biotechnol. 2015, 18, 147-154. [CrossRef]

74. Bernardo, R. Relationship between single-cross performance and molecular marker heterozygosity. Theor. Appl. Genet. 1992, 83, 628-634. [CrossRef] [PubMed]

75. Betran, F.; Beck, D.; Bänziger, M.; Edmeades, G. Genetic analysis of inbred and hybrid grain yield under stress and nonstress environments in tropical maize. Crop Sci. 2003, 43, 807-817. [CrossRef]

76. Parentoni, S.; Magalhães, J.; Pacheco, C.; Santos, M.; Abadie, T.; Gama, E.; Guimarães, P.; Meirelles, W.; Lopes, M.; Vasconcelos, M.; et al. Heterotic groups based on yield-specific combining ability data and phylogenetic relationship determined by RAPD markers for 28 tropical maize open pollinated varieties. Euphytica 2001, 121, 197-208. [CrossRef]

77. AOAC, C.A. Official Methods of Analysis of the Association of Analytical Chemists International; Official Methods: Gaithersburg, MD, USA, 2005.

78. Doyle, J. DNA Protocols for plants. In Molecular Techniques in Taxonomy; Springer Science and Business Media LLC: New York, NY, USA, 1991; pp. 283-293.

79. David, F.N.; Steel, R.G.D.; Torrie, J.H. Principles and Procedures of Statistics, A Biometrical Approach, 3rd ed.; McGraw Hill Inc. Book Co.: New York, NY, USA, 1997; pp. 352-358.

80. Griffing, B. Concept of general and specific combining ability in relation to diallel crossing systems. Aust. J. Biol. Sci. 1956, 9, 463-493. [CrossRef]

81. Zhang, Y.; Kang, M.S.; Lamkey, K.R. DIALLEL-SAS05: A comprehensive program for griffing's and gardner-eberhart analyses. Agron. J. 2005, 97, 1097-1106. [CrossRef] 
82. Wei, T.; Simko, V.; Levy, M.; Xie, Y.; Jin, Y.; Zemla, J. Corrplot: Visualization of a Correlation Matrix. Available online: https://github.com/taiyun/corrplot (accessed on 15 December 2019).

83. Jaccard, P. Nouvelles recherches sur la distribution florale. Bull. Soc. Vaud. Sci. Nat. 1908, 44, $223-270$.

(c)

(C) 2020 by the authors. Licensee MDPI, Basel, Switzerland. This article is an open access article distributed under the terms and conditions of the Creative Commons Attribution (CC BY) license (http://creativecommons.org/licenses/by/4.0/). 